\title{
An Asymmetric Synthesis of (+)-Isostrychnine Based on Catalytic Asymmetric Tandem Double Michael Addition
}

\author{
Pengyan Wang, ${ }^{+, \perp}$ Junhan Chen, ${ }^{\dagger, \perp}$ Weigang He, ${ }^{\dagger}$ Jiacheng Song, ${ }^{\dagger}$ Hengqian \\ Song, ${ }^{\dagger}$ Hongbo Wei, ${ }^{*, \dagger}$ and Weiqing $\mathrm{Xie}^{*, \dagger}, \|$
}

\footnotetext{
${ }^{\dagger}$ Shaanxi Key Laboratory of Natural Products \& Chemical Biology, College of Chemistry \& Pharmacy, Northwest A\&F University, 22 Xinong Road, Yangling 712100, Shaanxi, China

"Key Laboratory of Botanical Pesticide R\&D in Shaanxi Province, Yangling, Shaanxi 712100, China

*Email: weihb@nwafu.edu.cn; xiewq@nwafu.edu.cn.
}

\section{Contents}

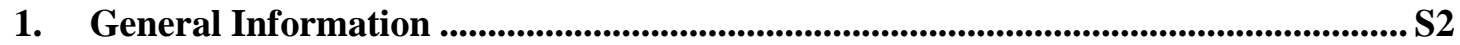

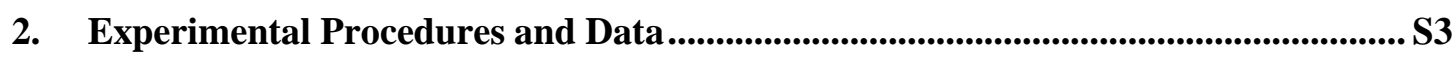

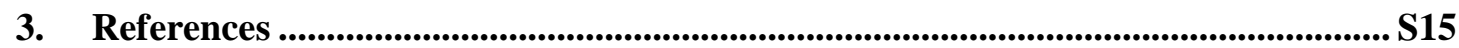

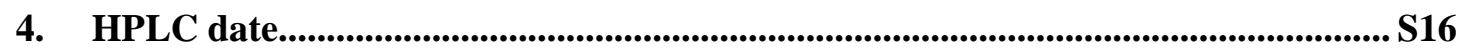

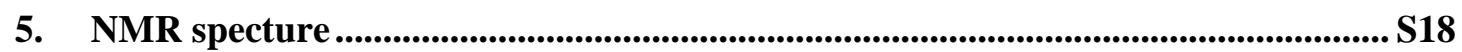




\section{General Information}

Unless otherwise noted, reagents were obtained from commercial sources and used without further purification. Non-aqueous reaction were conducted under an inert atmosphere of argon in flame-dried glassware. Anhydrous solvent were treated as follow: tetrahydrofuran and diethyl ether were distilled from sodium under argon atmosphere, dichloromethane and toluene was distilled from calcium hydride under argon atmosphere. Anhydrous 1,2-dichloroethane, chloroform, acetonitrile and ethyl acetate were commercial available. Thin layer chromatography was conducted on Merck 60 F254 pre-coated silica gel plates. Column chromatography was carried out by normal silica gel (40-60 $\mu \mathrm{m}, 200-400$ mesh, Silicycle P60). NMR data including ${ }^{1} \mathrm{H}$ NMR or ${ }^{13} \mathrm{C}$ NMR spectra were recorded on Bruker 500. ${ }^{1} \mathrm{H}$ NMR Chemical shifts were reported in ppm from the solvent resonance as the internal standard $\left(\mathrm{CDCl}_{3}\right.$ : 7.26 ppm; $\left(\mathrm{CD}_{3}\right)_{2} \mathrm{CO}: 2.05 \mathrm{ppm}$; $\left.\left(\mathrm{CD}_{3}\right)_{2} \mathrm{SO}: 2.50 \mathrm{ppm}\right) .{ }^{13} \mathrm{C}$ NMR chemical shifts were reported in ppm relative to the solvent $\left(\mathrm{CDCl}_{3}: 77.16 \mathrm{ppm} ;\left(\mathrm{CD}_{3}\right)_{2} \mathrm{CO}: 206.26\right.$ ppm; $\left.\left(\mathrm{CD}_{3}\right)_{2} \mathrm{SO}: 39.52 \mathrm{ppm}\right)$. Chiral HPLC analyses were performed on an Waters 2487 Series using a Daicel Chiralpak (AD-H, OD-H and IC) column with hexane/iPrOH as the eluent. Optical rotations were measured on Anton Paar MCP 300 polarimeter. High resolution massspectra (HRMS) were recorded on DIONEX UltiMate 3000 \& Bruker Compact TOF mass spectrometer. 


\section{Experimental Procedures and Data}<smiles>NCCc1c[nH]c2ccccc12</smiles>

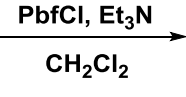

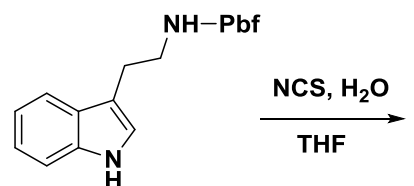

Pbf-tryptamine

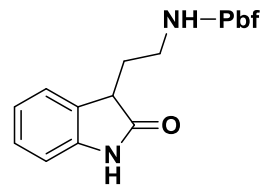

6

$\mathrm{Et}_{3} \mathrm{~N}$ (37 ml, $268 \mathrm{mmol}, 2.0$ equiv.) was added to a suspension of tryptamine (21.5 g, $134 \mathrm{mmol}, 1.0$ equiv.) in $500 \mathrm{~mL} \mathrm{CH}_{2} \mathrm{Cl}_{2}$. The mixture was cooled to $0{ }^{\circ} \mathrm{C}$, then $\mathrm{PbfCl}(40.3 \mathrm{~g}, 140 \mathrm{mmol}, 1.05$ equiv.) was added. The reaction mixture was stirred at this temperature for $3 \mathrm{~h}$. The resulting solution was diluted with $\mathrm{CH}_{2} \mathrm{Cl}_{2}$, washed with water and brine, and dried with anhydrous $\mathrm{Na}_{2} \mathrm{SO}_{4}$. The solvent was removed in vacuo and the residue was purified by column chromatography on silica gel $($ EtOAc/petroleum ether $=1: 2)$ to afford the products $(52.7 \mathrm{~g}, 127 \mathrm{mmol}, 95 \%)$ as a white amorphous solid.

${ }^{1}$ H NMR (500 MHz, DMSO-d $\left.{ }_{6}\right) \delta 10.81(\mathrm{~s}, 1 \mathrm{H}), 7.36(\mathrm{~d}, J=6.0 \mathrm{~Hz}, 1 \mathrm{H}), 7.31(\mathrm{~d}, J$ $=8.2 \mathrm{~Hz}, 1 \mathrm{H}), 7.25(\mathrm{~d}, J=7.9 \mathrm{~Hz}, 1 \mathrm{H}), 7.08(\mathrm{~s}, 1 \mathrm{H}), 7.03(\mathrm{t}, J=7.7 \mathrm{~Hz}, 1 \mathrm{H}), 6.91(\mathrm{t}$, $J=7.7 \mathrm{~Hz}, 1 \mathrm{H}), 3.00(\mathrm{t}, J=7.3 \mathrm{~Hz}, 2 \mathrm{H}), 2.95(\mathrm{~s}, 2 \mathrm{H}), 2.77(\mathrm{~d}, J=7.9 \mathrm{~Hz}, 2 \mathrm{H}), 2.42$ $(\mathrm{d}, J=15.9 \mathrm{~Hz}, 6 \mathrm{H}), 2.01(\mathrm{~s}, 3 \mathrm{H}), 1.40(\mathrm{~s}, 6 \mathrm{H})$.

${ }^{13}$ C NMR (126 MHz, DMSO-d $\left.{ }_{6}\right) \delta 158.5,138.4,136.2,133.1,129.3,126.9,125.0$, $122.9,120.9,118.2,117.8,116.9,111.4,111.0,86.7,42.6,42.4,28.2$, 25.2 , $19.1,17.4,12.3$

HRMS (ESI) m/z: [M + H] $]^{+}$Calcd for $\mathrm{C}_{23} \mathrm{H}_{29} \mathrm{~N}_{2} \mathrm{O}_{3} \mathrm{~S}$ 413.1893; Found 413.1888.

Water $(0.54 \mathrm{~mL}, 30 \mathrm{mmol}, 1.5$ equiv. $)$ was added to a solution of $\mathrm{Pbf}$ protected tryptamine ( $8.5 \mathrm{~g}, 20 \mathrm{mmol}, 1$ equiv.) in $200 \mathrm{~mL}$ THF and the solution was cooled to $-20{ }^{\circ} \mathrm{C} . \mathrm{N}$-Chlorosuccinimide $(2.8 \mathrm{~g}, 21 \mathrm{mmol}, 1.05$ equiv.) was added, and the mixture was stirred at $-20{ }^{\circ} \mathrm{C}$ for $1 \mathrm{~h}$, then moved to an ice bath for $1 \mathrm{~h}$ and finally stirred at room temperature overnight. The solvent was removed in vacuo and the residue was diluted with $\mathrm{CH}_{2} \mathrm{Cl}_{2}$, washed with water and brine, and dried over anhydrous $\mathrm{Na}_{2} \mathrm{SO}_{4}$. The solvent was removed in vacuo to provide crude product. 
Trituration/washing with EtOAc provided pure product 6 as a white amorphous solid(6.8 g, $16 \mathrm{mmol}, 80 \%)$.

${ }^{1}$ H NMR (500 MHz, DMSO-d $) \delta 10.36(\mathrm{~s}, 1 \mathrm{H}), 7.42(\mathrm{~s}, 1 \mathrm{H}), 7.14(\mathrm{t}, J=7.0 \mathrm{~Hz}$, 1H), 6.99 (d, $J=7.4 \mathrm{~Hz}, 1 \mathrm{H}), 6.89$ (t, $J=6.3 \mathrm{~Hz}, 1 \mathrm{H}), 6.79$ (d, $J=7.6 \mathrm{~Hz}, 1 \mathrm{H}), 3.36$ (m, 1H), $2.98(\mathrm{~s}, 2 \mathrm{H}), 2.94-2.78(\mathrm{~m}, 2 \mathrm{H}), 2.44(\mathrm{~s}, 3 \mathrm{H}), 2.37$ (s, 3H), $2.02(\mathrm{~s}, 3 \mathrm{H})$, $1.87(\mathrm{t}, J=7.3 \mathrm{~Hz}, 1 \mathrm{H}), 1.82-1.71(\mathrm{t}, 1 \mathrm{H}), 1.41(\mathrm{~s}, 6 \mathrm{H})$.

${ }^{13}$ C NMR (126 MHz, DMSO-d $\left.\boldsymbol{d}_{6}\right) \delta 178.6,158.6,142.5,138.5,133.3,129.2$, $127.7,125.1,123.7,121.2,116.9,109.3,86.8,42.7,42.4,38.9,30.3,28.2$, $19.0,17.4,12.3$.

HRMS (ESI) m/z: [M + H] $]^{+}$Calcd for $\mathrm{C}_{23} \mathrm{H}_{29} \mathrm{~N}_{2} \mathrm{O}_{4} \mathrm{~S}$ 429.1843; Found 429.1849.
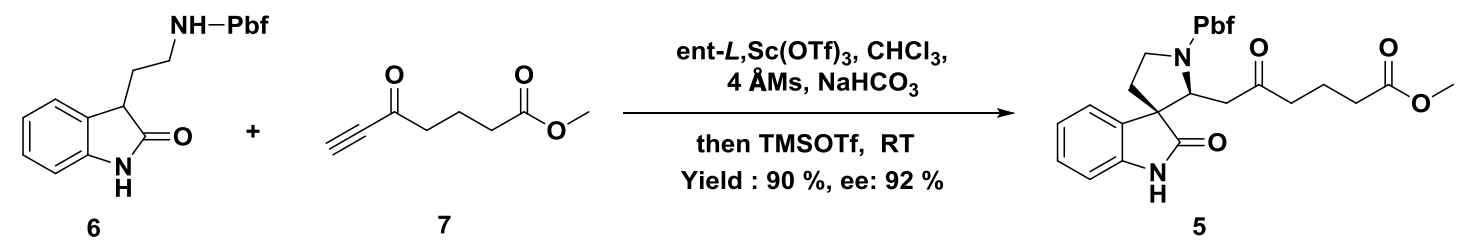

N,N'-dioxide ligand ent- $L$ (0.81 g, $1.4 \mathrm{mmol}, 0.048$ equiv.), scandium triflate (0.59 g, 1.2 mmol, 0.04 equiv.), and $4 \AA$ molecular sieves (6.00 g) were stirred in 50 $\mathrm{mL} \mathrm{CH}_{2} \mathrm{Cl}_{2}$ at room temperature for 2 hours ${ }^{[1]}$. After that the solvent was removed in vacuo and $300 \mathrm{~mL} \mathrm{CHCl}_{3}$ was added. Oxindole 6 (12.8 g, $30.0 \mathrm{mmol}, 1.0$ equiv.), alkynones 7 (5.52 g, 33.0 mmol, 1.2 equiv.), $\mathrm{NaHCO}_{3}$ (2,52 g, 30.0 mmol, 1.0 equiv) was added to the suspension and the mixture was stirred at $40{ }^{\circ} \mathrm{C}$ in oil bath for $48 \mathrm{~h}$ until the oxindole 6 was consumed. the reaction was moved to room temperature and TMSOTf ( $0.55 \mathrm{~mL}, 3 \mathrm{mmol}, 0.1$ equiv.) was added portionwise. the reaction mixture was stirred for final $1 \mathrm{~h}$, The resulting mixture was filtrated through Celite pad and diluted with $\mathrm{CH}_{2} \mathrm{Cl}_{2}$, washed with water. The aqueous phase was extracted with $\mathrm{CH}_{2} \mathrm{Cl}_{2}$ (3 x $\left.150 \mathrm{ml}\right)$. The combined organic extracts were washed with brine, dried over anhydrous $\mathrm{Na}_{2} \mathrm{SO}_{4}$ and concentrated in vacuo. The residue was purified by column chromatography on silica gel (EtOAc/petroleum ether $=1: 2)$ to afford the products $5(15.7 \mathrm{~g}, 27 \mathrm{mmol}, 90 \%)$ as a white amorphous solid and its diastereomer 5' 
$(0.62 \mathrm{~g})$.

Enantiomeric excess was found to be $93.7 \%$ by chiral HPLC (ChiralPak AD-H

column, $n$-hexane $/ i-\mathrm{PrOH}=70 / 30$, flow rate $=0.5 \mathrm{~mL} / \mathrm{min}, \mathrm{I}=214 \mathrm{~nm}) \mathrm{t}_{\mathrm{R}}=23.7 \mathrm{~min}$ (major), $20.5 \mathrm{~min}$ (minor).

$[\alpha]_{\mathrm{D}}^{20}=-44.7\left(c 0.34, \mathrm{CHCl}_{3}\right)$

${ }^{1}$ H NMR (500 MHz, Chloroform-d) $\delta 8.16(\mathrm{~s}, 1 \mathrm{H}), 7.25(\mathrm{~d}, J=5.7 \mathrm{~Hz}, 1 \mathrm{H}), 7.13(\mathrm{t}$, $J=7.7 \mathrm{~Hz}, 1 \mathrm{H}), 6.97(\mathrm{t}, J=7.6 \mathrm{~Hz}, 1 \mathrm{H}), 6.75(\mathrm{~d}, J=7.7 \mathrm{~Hz}, 1 \mathrm{H}), 4.62(\mathrm{dd}, J=11.0$, $3.3 \mathrm{~Hz}, 1 \mathrm{H}), 3.67-3.60(\mathrm{~m}, 4 \mathrm{H}), 3.48-3.52(\mathrm{~m}, 1 \mathrm{H}), 3.38(\mathrm{dd}, J=18.0,11.0 \mathrm{~Hz}, 1 \mathrm{H})$, 2.99 (s, 2H), $2.88(\mathrm{dd}, J=18.0,3.3 \mathrm{~Hz}, 1 \mathrm{H}), 2.60$ (s, 3H), 2.53 (s, 3H), $2.46-2.29$ (m, $3 \mathrm{H}), 2.23(\mathrm{td}, J=7.3,2.0 \mathrm{~Hz}, 2 \mathrm{H}), 2.13(\mathrm{~s}, 3 \mathrm{H}), 2.01-1.97(\mathrm{~m}, 1 \mathrm{H}), 1.76-1.72(\mathrm{~m}, 2 \mathrm{H})$, $1.48(\mathrm{~d}, 6 \mathrm{H})$.

${ }^{13}$ C NMR (126 MHz, Chloroform-d) $\delta$ 208.7 , 178.2 , 173.8 , 160.4 , 140.9 , 139.6, $135.1,134.0$, $128.4,127.0,125.5$, 123.5 , 122.9 , 118.4 , 109.8 , 87.1 , 62.0 , 55.2 , $51.6,46.6,44.7,43.3$, $41.3,36.1,33.0,28.7$, $28.6,19.8,18.6,18.1,12.7$.

HRMS (ESI) m/z: [M+ H] $]^{+}$Calcd for $\mathrm{C}_{31} \mathrm{H}_{39} \mathrm{~N}_{2} \mathrm{O}_{7} \mathrm{~S}$ 583.2472; Found 583.2474.

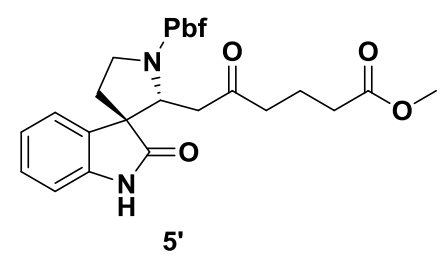

$[\alpha]_{\mathrm{D}}^{20}=-1.76\left(c 0.58, \mathrm{CHCl}_{3}\right)$

${ }^{1}$ H NMR (500 MHz, Chloroform-d) $\delta 8.40(\mathrm{~s}, 1 \mathrm{H}), 7.26(\mathrm{t}, J=7.7 \mathrm{~Hz}, 1 \mathrm{H}), 7.21(\mathrm{~d}$, $J=7.5 \mathrm{~Hz}, 1 \mathrm{H}), 7.05$ (t, $J=7.6 \mathrm{~Hz}, 1 \mathrm{H}), 6.93(\mathrm{~d}, J=7.9 \mathrm{~Hz}, 1 \mathrm{H}), 4.79$ (dd, $J=10.3$, $4.5 \mathrm{~Hz}, 1 \mathrm{H}), 3.71-3.66(\mathrm{~m}, 1 \mathrm{H}), 3.65(\mathrm{~s}, 3 \mathrm{H}), 3.46-3.40(\mathrm{~m}, 1 \mathrm{H}), 3.24(\mathrm{dd}, J=17.7,4.6$ $\mathrm{Hz}, 1 \mathrm{H}), 3.04(\mathrm{~s}, 2 \mathrm{H}), 2.79-2.70(\mathrm{~m}, 1 \mathrm{H}), 2.69(\mathrm{~s}, 3 \mathrm{H}), 2.61(\mathrm{~s}, 3 \mathrm{H}), 2.55-2.45(\mathrm{~m}$, 1H), $2.19(\mathrm{~s}, 3 \mathrm{H}), 2.17-2.12(\mathrm{~m}, 1 \mathrm{H}) 2.10-1.99(\mathrm{~m}, 2 \mathrm{H}), 1.95-1.88(\mathrm{~m}, 1 \mathrm{H}), 1.76-1.70$ (m, 1H), 1.58-1.45 (m, 8H).

${ }^{13}$ C NMR (126 MHz, Chloroform-d) $\delta$ 206.6 , 179.2 , 141.4 , 140.8 , 135.1 , 130.0 , $128.8,127.1,125.4,124.5,121.9,118.3,110.5,87.0,61.0,57.4,51.4$, 47.6 , $45.3,43.2,41.1,37.1,32.8,28.6,19.5,18.3,17.8,12.5$. 
HRMS (ESI) m/z: [M + H] $]^{+}$Calcd for $\mathrm{C}_{31} \mathrm{H}_{39} \mathrm{~N}_{2} \mathrm{O}_{7} \mathrm{~S}$ 583.2472; Found 583.2476.<smiles>COC(=O)CCCC(=O)CC1N(c2ccccc2)CC[C@]12C(=O)Nc1ccccc12</smiles>

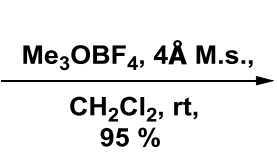

5

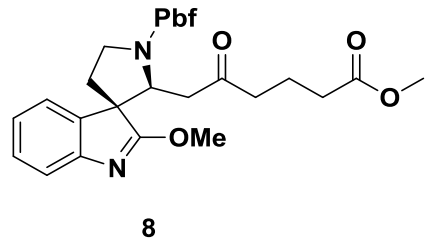

$\mathrm{Me}_{3} \mathrm{O} \cdot \mathrm{BF}_{4}(3.85 \mathrm{~g}, 15 \mathrm{mmol}, 1.5$ equiv. $)$ and $4 \AA$ molecular sieves $(2 \mathrm{~g})$ were added to a solution of 5 (5.9 g, $10 \mathrm{mmol}, 1.0$ equiv.) in $100 \mathrm{~mL} \mathrm{CH}_{2} \mathrm{Cl}_{2}$ and the mixture was stirred at room temperature overnight. After the starting material disappeared (monitored by TLC), The mixture was filtrated through Celite pad and diluted with $\mathrm{CH}_{2} \mathrm{Cl}_{2}$. The filtrate was washed with water. The aqueous phase was extracted with $\mathrm{CH}_{2} \mathrm{Cl}_{2}(3 \times 100 \mathrm{ml})$. The combined organic extracts were washed with brine and dried over anhydrous $\mathrm{Na}_{2} \mathrm{SO}_{4}$. The solvent was removed in vacuo to afford products 8 (5.8 g, $9.6 \mathrm{mmol}, 95 \%)$ as a white amorphous solid and the residue was used directly in the following step without further purification.

$[\alpha]_{\mathrm{D}}^{20}=-52.4\left(c 0.43, \mathrm{CHCl}_{3}\right)$

${ }^{1}$ H NMR (500 MHz, Chloroform-d) $\delta 7.29(\mathrm{~d}, J=7.7 \mathrm{~Hz}, 1 \mathrm{H}), 7.23(\mathrm{t}, J=7.7 \mathrm{~Hz}$, 1H), $7.17(\mathrm{~d}, J=7.4 \mathrm{~Hz}, 1 \mathrm{H}), 7.03(\mathrm{t}, J=7.4 \mathrm{~Hz}, 1 \mathrm{H}), 4.65(\mathrm{dd}, J=9.0,4.2 \mathrm{~Hz}, 1 \mathrm{H})$, $3.98(\mathrm{~s}, 3 \mathrm{H}), 3.66-3.60(\mathrm{~m}, 4 \mathrm{H}), 3.51-3.46(\mathrm{~m}, 1 \mathrm{H}), 2.98(\mathrm{~s}, 2 \mathrm{H}), 2.91(\mathrm{dd}, J=17.6$, $4.2 \mathrm{~Hz}, 1 \mathrm{H}), 2.67$ (dd, $J=17.6,9.0 \mathrm{~Hz}, 1 \mathrm{H}), 2.58(\mathrm{~s}, 3 \mathrm{H}), 2.51(\mathrm{~s}, 3 \mathrm{H}), 2.29-2.24(\mathrm{~m}$, 3H), 2.22-2.15 (m, 2H), $2.13(\mathrm{~s}, 3 \mathrm{H}), 2.10-2.02(\mathrm{~m}, 1 \mathrm{H}), 1.68(\mathrm{p}, J=7.2 \mathrm{~Hz}, 2 \mathrm{H})$, $1.47(\mathrm{~s}, 6 \mathrm{H})$.

${ }^{13}$ C NMR (126 MHz, Chloroform-d) $\delta$ 205.9 , $179.8,173.6,160.3,151.5,140.8$, $138.9,135.1,128.7,127.1,125.5,124.3,122.1,118.7,118.4,87.1,60.4,60.1$, $56.6,51.6,46.5$, 44.9 , 43.3 , 41.2 , 33.8 , 32.9 , 28.6, 28.6, $19.7,18.4,18.0,12.7$. HRMS (ESI) m/z: $[\mathrm{M}+\mathrm{H}]^{+}$Calcd for $\mathrm{C}_{32} \mathrm{H}_{41} \mathrm{~N}_{2} \mathrm{O}_{7} \mathrm{~S}$ 597.2629; Found 597.2636. 


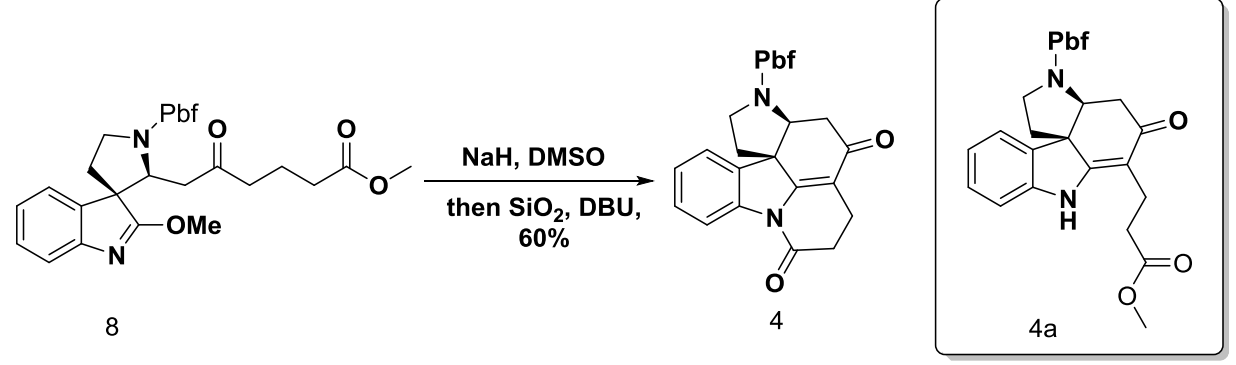

$\mathrm{NaH}(0.24 \mathrm{~g}, 6 \mathrm{mmol}, 2.0$ equiv.) in $20 \mathrm{~mL}$ DMSO was stirred in room temperature for $2 \mathrm{~h}$ under Ar atmosphere, then 8 (1.78 g, $3 \mathrm{mmol}, 1.0$ equiv.) in 10 $\mathrm{mL}$ DMSO was added to the solution via syringe. The reaction was stirred for $10 \mathrm{~min}$ until starting material disappeared(monitored by TLC)and afforded $4 \mathrm{a}$. $\mathrm{SiO}_{2}(2 \mathrm{~g}$ ) was added to the mixture and stirred for $30 \mathrm{~min}$. Finally DBU(45 mg, $0.3 \mathrm{mmol}, 0.1$ equiv.) was added and stirred for another $1 \mathrm{~h}$. The resulting solution was diluted with $100 \mathrm{ml}$ EtOAc, washed with $150 \mathrm{ml}$ water. The aqueous phase was extracted with EtOAc $(3 \mathrm{x}$ $100 \mathrm{ml})$. The combined organic extracts were washed with water and brine, dried over anhydrous $\mathrm{Na}_{2} \mathrm{SO}_{4}$ and concentrated in vacuo. The resulting oil was purified via flash column chromatography on silica gel $($ EtOAc/petroleum ether $=1: 5)$ to afford the products 4(0.96 g, $1.8 \mathrm{mmol}, 60 \%)$ as a white amorphous solid.

The products 4 (5.2 g) was stirred in $200 \mathrm{~mL}$ EtOAc for $2 \mathrm{~h}$ and filtered through filter paper. Then the organic phase was concentrated in vacuo afforded 4(4.6 g). The enantiomeric excess of 4 was improved from $93.7 \%$ to $97.0 \%$.

Enantiomeric excess was found to be $97.0 \%$ by chiral HPLC (ChiralPak OD-H column, $n$-hexane $/ i$-PrOH $=70 / 30$, flow rate $=0.7 \mathrm{~mL} / \mathrm{min}, \mathrm{I}=214 \mathrm{~nm}) \mathrm{t}_{\mathrm{R}}=40.3 \mathrm{~min}$ (major), $32.3 \mathrm{~min}$ (minor).

$[\alpha]_{\mathrm{D}}^{20}=-218.5\left(c 0.29, \mathrm{CHCl}_{3}\right)$

${ }^{1}$ H NMR (500 MHz, Chloroform-d) $\delta 8.18(\mathrm{~d}, J=8.1 \mathrm{~Hz}, 1 \mathrm{H}), 7.60(\mathrm{~d}, J=7.6 \mathrm{~Hz}$, $1 \mathrm{H}), 7.35$ (t, $J=7.8 \mathrm{~Hz}, 1 \mathrm{H}), 7.17(\mathrm{t}, J=7.6 \mathrm{~Hz}, 1 \mathrm{H}), 4.62-4.52(\mathrm{~m}, 1 \mathrm{H}), 3.77(\mathrm{dd}, J=$ 10.1, 4.1 Hz, 2H), 3.00 (s, 2H), 2.90-2.82 (m, 2H), 2.81-2.69 (m, 2H), 2.63-2.54 (m, 5H), $2.52(\mathrm{~s}, 3 \mathrm{H}), 2.37-2.29(\mathrm{~m}, 1 \mathrm{H}), 2.17-2.10(\mathrm{~m}, 4 \mathrm{H}), 1.51(\mathrm{~s}, 3 \mathrm{H}), 1.49(\mathrm{~s}, 3 \mathrm{H})$. 
${ }^{13}$ C NMR (126 MHz, Chloroform-d) $\delta$ 192.2 , 167.3 , 160.4 , 159.6 , 140.6 , 139.1, $134.8,134.0$, $129.3,127.7,125.6,125.6,123.4,118.6,115.8,113.3$, 87.2 , 61.7 , $52.1,45.2,43.3,42.1,40.3,32.3,28.7,28.7,19.9,18.3,17.9,12.7$.

HRMS (ESI) m/z: [M + H] $]^{+}$Calcd for $\mathrm{C}_{30} \mathrm{H}_{33} \mathrm{~N}_{2} \mathrm{O}_{5} \mathrm{~S}$ 533.2105; Found 533.2114.

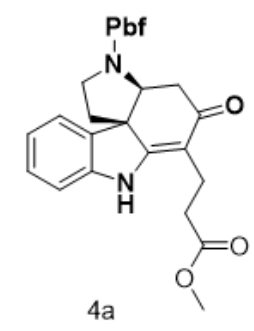

$[\alpha]_{\mathrm{D}}^{20}=-256.3\left(c 0.32, \mathrm{CHCl}_{3}\right)$

${ }^{1}$ H NMR (500 MHz, Chloroform-d) $\delta 8.48(\mathrm{~s}, 1 \mathrm{H}), 7.55(\mathrm{~d}, J=7.5 \mathrm{~Hz}, 1 \mathrm{H}), 7.23(\mathrm{~d}$, $J=7.9 \mathrm{~Hz}, 1 \mathrm{H}), 6.96(\mathrm{~d}, J=7.3 \mathrm{~Hz}, 2 \mathrm{H}), 4.43(\mathrm{t}, J=8.1 \mathrm{~Hz}, 1 \mathrm{H}), 3.79-3.71(\mathrm{~m}, 2 \mathrm{H})$, $3.63(\mathrm{~s}, 3 \mathrm{H}), 2.99(\mathrm{~s}, 2 \mathrm{H}), 2.75-2.67(\mathrm{~m}, 2 \mathrm{H}), 2.59$ (s, 3H), 2.54-2.49 (m, 5H), 2.47-2.40 (m, 3H), 2.13-2.05 (m, 4H), $1.49(\mathrm{~d}, J=8.9 \mathrm{~Hz}, 6 \mathrm{H})$.

${ }^{13}$ C NMR (126 MHz, Chloroform-d) $\delta$ 191.7 , 176.4 , 167.0 , 142.9 , 134.5 , 128.7 , $125.4,123.5,122.0,118.3,110.1,109.0,87.0,59.7,53.8,52.0$, 44.9 , 43.3 , $41.4,41.2,33.0,28.6,19.7,19.0,18.2,12.6$.

HRMS (ESI) m/z: [M + H] $]^{+}$Calcd for $\mathrm{C}_{31} \mathrm{H}_{36} \mathrm{~N}_{2} \mathrm{O}_{6} \mathrm{~S}$ 565.2367; Found 565.2373.

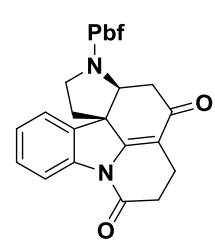

4

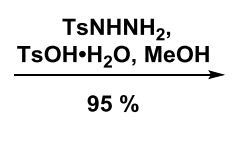

$95 \%$

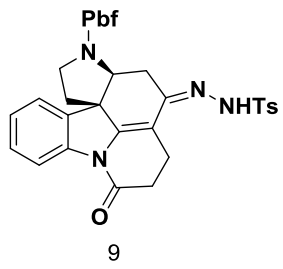

9

$\mathrm{TsNHNH}_{2}(0.57 \mathrm{~g}, 3.1 \mathrm{mmol}, 1.1$ equiv. $)$ and TsOH$\cdot \mathrm{H}_{2} \mathrm{O}(48 \mathrm{mg}, 0.28 \mathrm{mmol}, 0.1$ equiv.) were added to a solution of $4(1.5 \mathrm{~g}, 2.8 \mathrm{mmol}, 1.0$ equiv.) in $30 \mathrm{ml} \mathrm{MeOH}$. The solution was heated to $70{ }^{\circ} \mathrm{C}$ in oil bath for $12 \mathrm{~h}$. The resulting reaction was filtered through filter paper and washed with $\mathrm{MeOH}$ to afford products 9 as a white amorphous solid. The residue $9(1.86 \mathrm{~g}, 2.6 \mathrm{mmol}, 95 \%)$ was used directly in following step without further purification. $[\alpha]_{\mathrm{D}}{ }^{20}=-132.4\left(c 0.46, \mathrm{CHCl}_{3}\right)$ 
${ }^{1}$ H NMR (500 MHz, Chloroform-d) $\delta 8.14(\mathrm{~d}, J=8.1 \mathrm{~Hz}, 1 \mathrm{H}), 7.86(\mathrm{~d}, J=7.1 \mathrm{~Hz}$, 2H), $7.52(\mathrm{~s}, 1 \mathrm{H}), 7.32(\mathrm{~d}, J=8.1 \mathrm{~Hz}, 2 \mathrm{H}), 7.28(\mathrm{t}, J=8.0 \mathrm{~Hz}, 1 \mathrm{H}), 7.11(\mathrm{~d}, J=7.5$ $\mathrm{Hz}, 1 \mathrm{H}), 7.00(\mathrm{t}, J=7.6 \mathrm{~Hz}, 1 \mathrm{H}), 4.30$ (t, $J=8.0 \mathrm{~Hz}, 1 \mathrm{H}), 3.70$ (t, $J=9.5 \mathrm{~Hz}, 1 \mathrm{H})$, $3.39(\mathrm{q}, J=9.2 \mathrm{~Hz}, 1 \mathrm{H}), 3.12(\mathrm{dd}, J=17.6,7.3 \mathrm{~Hz}, 1 \mathrm{H}), 3.04-2.93(\mathrm{~m}, 3 \mathrm{H}), 2.73(\mathrm{dd}$, $J=11.9,8.0 \mathrm{~Hz}, 2 \mathrm{H}), 2.58(\mathrm{~s}, 3 \mathrm{H}), 2.53(\mathrm{~s}, 3 \mathrm{H}), 2.49-2.44(\mathrm{~m}, 1 \mathrm{H}), 2.43(\mathrm{~s}, 3 \mathrm{H}), 2.33$ $(\mathrm{q}, J=10.7 \mathrm{~Hz}, 1 \mathrm{H}), 2.29-2.21(\mathrm{~m}, 1 \mathrm{H}), 2.16(\mathrm{~s}, 3 \mathrm{H}), 2.00(\mathrm{dd}, J=12.6,7.4 \mathrm{~Hz}, 1 \mathrm{H})$, $1.52(\mathrm{~s}, 3 \mathrm{H}), 1.51(\mathrm{~s}, 3 \mathrm{H})$.

${ }^{13}$ C NMR (126 MHz, Chloroform-d) $\delta$ 167.2 , 160.3 , 148.2 , 145.3 , 144.0 , 140.4 , $139.3,135.4,134.8,134.2,129.5,129.0,128.2$, $127.1,125.6,124.7,122.7$, 118.5 , 115.7 , 111.3 , 87.2 , 61.3 , 50.9 , 44.2 , 43.2 , 39.1 , 32.4 , 29.4 , 28.6 , 21.6, $19.9,19.1,18.5,12.6$.

HRMS (ESI) m/z: $[\mathrm{M}+\mathrm{H}]^{+}$Calcd for $\mathrm{C}_{37} \mathrm{H}_{41} \mathrm{~N}_{4} \mathrm{O}_{6} \mathrm{~S}_{2}$ 701.2462; Found 701.2467.
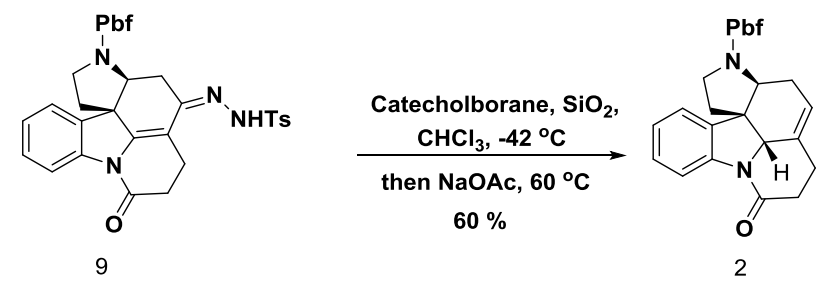

Catecholborane (1.32 g, $11 \mathrm{mmol}, 6.0$ equiv.) in $10 \mathrm{ml} \mathrm{CHCl}_{3}$ was added dropwise to a mixture of hydrazone 9 (1.3 g, $1.8 \mathrm{mmol}, 1.0$ equiv.) and silica gel ( $2 \mathrm{~g})$ in $20 \mathrm{ml} \mathrm{CHCl}{ }_{3}$ at $-42{ }^{\circ} \mathrm{C}$. After $1 \mathrm{~h}$ at $-42{ }^{\circ} \mathrm{C}, \mathrm{NaOAc} \cdot 3 \mathrm{H}_{2} \mathrm{O}(490 \mathrm{mg}, 3.6 \mathrm{mmol}, 2.0$ equiv.) was added and the reaction mixture was heated under reflux in oil bath for 16 h. The reaction mixture was poured into water and isolated by extractive work-up ${ }^{[2]}$. The crude material was purified by flash chromatography over silica gel $($ EtOAc/petroleum ether $=1: 3)$ to give $2(568 \mathrm{mg}, 1.1 \mathrm{mmol}, 60 \%)$ as a white amorphous solid.

$[\alpha]_{\mathrm{D}}^{20}=-4.5\left(c 0.45, \mathrm{CHCl}_{3}\right)$

${ }^{1}$ H NMR (500 MHz, Chloroform-d) $\delta 8.14(\mathrm{~d}, J=8.0 \mathrm{~Hz}, 1 \mathrm{H}), 7.24(\mathrm{~d}, J=7.0 \mathrm{~Hz}$, 1H), 6.96-6.90 (m, 2H), $5.67(\mathrm{~d}, J=6.3 \mathrm{~Hz}, 1 \mathrm{H}), 4.35(\mathrm{~s}, 1 \mathrm{H}), 3.80(\mathrm{dd}, J=9.1,5.9$ $\mathrm{Hz}, 1 \mathrm{H}), 3.65(\mathrm{td}, J=9.7,3.6 \mathrm{~Hz}, 1 \mathrm{H}), 3.43(\mathrm{q}, J=8.7 \mathrm{~Hz}, 1 \mathrm{H}), 2.99(\mathrm{~s}, 2 \mathrm{H})$, 
2.73-2.64 (m, 3H), 2.61-2.53 (m, 2H), $2.43(\mathrm{~s}, 6 \mathrm{H}), 2.32-2.22(\mathrm{~m}, 3 \mathrm{H}), 2.12(\mathrm{~s}, 3 \mathrm{H})$, $1.51(\mathrm{~s}, 3 \mathrm{H}), 1.49$ (s, 3H).

${ }^{13}$ C NMR (126 MHz, Chloroform-d) $\delta$ 169.0 , 160.1 , 140.8 , 140.7 , 135.8, 134.8 , 130.9 , 128.9 , 127.9 , 125.3 , 124.2 , 123.9 , 120.2 , 118.2 , 116.4 , 87.0 , 63.5 , 61.2 , $51.9,43.5,43.4,36.3,33.2,28.7,27.8,27.3,19.9,18.4,12.7$.

HRMS (ESI) m/z: $[\mathrm{M}+\mathrm{H}]^{+}$Calcd for $\mathrm{C}_{30} \mathrm{H}_{35} \mathrm{~N}_{2} \mathrm{O}_{4} \mathrm{~S}$ 519.2312; Found 519.2318.
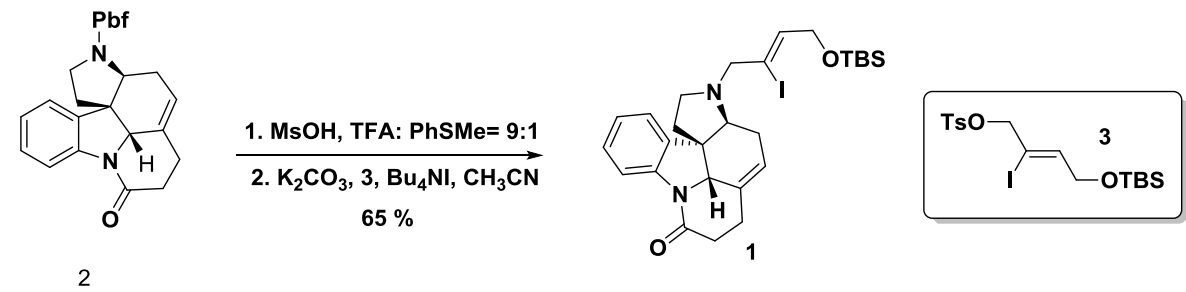

\section{Step 1}

$\mathrm{MsOH}(135 \mu \mathrm{L}, 2.1 \mathrm{mmol}, 2.7$ equiv.) was added to a mixture of 2(400 mg, 0.77 mmol, 1.0 equiv) in $10 \mathrm{~mL}$ TFA and $1.1 \mathrm{~mL} \mathrm{PhSMe}$. The reaction was stirred at room temperature for $1.5 \mathrm{~h}$. The resulting solution was concentrated in vacuo, diluted with $\mathrm{CH}_{2} \mathrm{Cl}_{2}$ and basified with saturated $\mathrm{NaHCO}_{3}$ solution. The mixture was extracted with $\mathrm{CH}_{2} \mathrm{Cl}_{2}(5 \times 30 \mathrm{~mL})$ and the combined organic extracts were washed with brine, dried over anhydrous $\mathrm{Na}_{2} \mathrm{SO}_{4}$ and concentrated in vacuo. According the report of Reissig ${ }^{[3]}$, Column chromatography on $\mathrm{SiO}_{2}$ always led, due to decomposition, to substantially losses of the product, the crude products was used directly in following step without further purification.

\section{Step 2}

The crude product was dissolved in $\mathrm{CH}_{3} \mathrm{CN}(10 \mathrm{~mL})$ and $n \mathrm{Bu}_{4} \mathrm{NI}(144 \mathrm{mg}, 0.39$ mmol, 0.5 equiv.), $\mathrm{K}_{2} \mathrm{CO}_{3}$ (224mg, $1.6 \mathrm{mmol}, 2.1$ equiv) and vinyl iodide 3 (371 mg, $0.77 \mathrm{mmol}, 1.0$ equiv.) were added. The reaction was stirred overnight, the solid removed by filtration and the solvent evaporated. The residue was purified by column chromatography on $\mathrm{SiO}_{2}($ EtOAc/petroleum ether = 1: 5$)$ affording product $\mathbf{1}(288$ $\mathrm{mg}, 0.5 \mathrm{mmol}, 65 \%$ over 2 steps) as a colorless oil ${ }^{[3]}$. 
$[\alpha]_{\mathrm{D}}^{20}=-56.6\left(c 0.75, \mathrm{CHCl}_{3}\right)$

${ }^{1}$ H NMR (500 MHz, Chloroform- $d$ ) $\delta 8.11(\mathrm{~d}, J=8.0 \mathrm{~Hz}, 1 \mathrm{H}), 7.69$ (d, $J=7.5 \mathrm{~Hz}$, 1H), 7.26-7.20 (t, 1H), $7.08(\mathrm{t}, J=7.5 \mathrm{~Hz}, 1 \mathrm{H}), 6.09$ (t, $J=5.1 \mathrm{~Hz}, 1 \mathrm{H}), 5.68(\mathrm{~d}, J=$ $7.1 \mathrm{~Hz}, 1 \mathrm{H}), 4.32(\mathrm{~s}, 1 \mathrm{H}), 4.25(\mathrm{~d}, J=4.9 \mathrm{~Hz}, 2 \mathrm{H}), 3.54(\mathrm{~d}, J=14.0 \mathrm{~Hz}, 1 \mathrm{H}), 3.43(\mathrm{~d}$, $J=14.0 \mathrm{~Hz}, 1 \mathrm{H}), 3.21-3.17(\mathrm{~m}, 1 \mathrm{H}), 2.89(\mathrm{dd}, J=8.8,5.5 \mathrm{~Hz}, 1 \mathrm{H}), 2.78-2.61(\mathrm{~m}, 3 \mathrm{H})$, 2.59-2.50 (m, 2H), $2.20(\mathrm{~m}, 1 \mathrm{H}), 2.17-1.96(\mathrm{~m}, 3 \mathrm{H}), 0.90(\mathrm{~s}, 9 \mathrm{H}), 0.08(\mathrm{~s}, 6 \mathrm{H})$.

${ }^{13}$ C NMR (126 MHz, Chloroform-d) $\delta$ 169.1 , 140.5 , 138.2 , 136.8 , 131.4 , 128.1 , $125.6,124.4,120.1,115.9,105.3,68.1,64.2,63.0,62.0,52.0,47.9$, 37.1 , 33.2 , $27.4,26.0,22.2,18.4,-5.0$.

HRMS (ESI) m/z: $[\mathrm{M}+\mathrm{H}]^{+}$Calcd for $\mathrm{C}_{27} \mathrm{H}_{38} \mathrm{IN}_{2} \mathrm{O}_{2} \mathrm{Si}$ 577.1742; Found 577.1742.
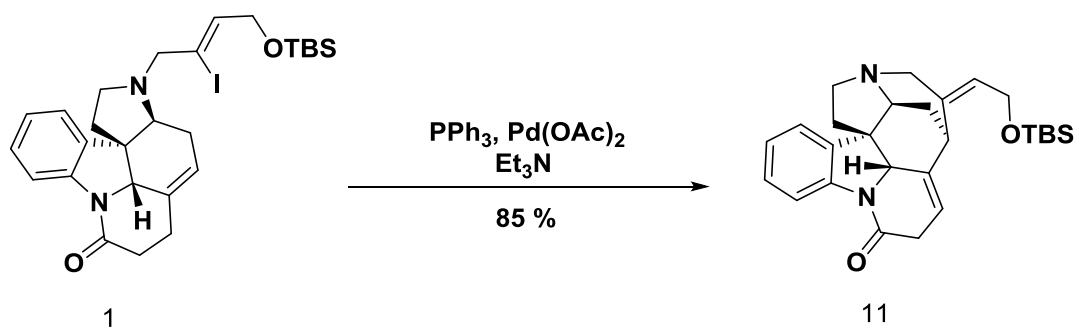

A solution of amine $1(75 \mathrm{mg}, 0.13 \mathrm{mmol}, 1.0$ equiv. $), \mathrm{Pd}(\mathrm{OAc})_{2}(5.6 \mathrm{mg}, 0.026$ mmol, 0.2 equiv.), $\mathrm{PPh}_{3}\left(13 \mathrm{mg}, 0.052 \mathrm{mmol}, 0.4\right.$ equiv.) in $5 \mathrm{~mL} \mathrm{Et}_{3} \mathrm{~N}$ was stirred at room temperature for $1 \mathrm{~h}$. Then the reaction was heated in $65^{\circ} \mathrm{C}$ in oil bath for $3 \mathrm{~h}$. After cooling to room temperature, the reaction was concentrated in vacuo, diluted with $\mathrm{CH}_{2} \mathrm{Cl}_{2}$ and washed with saturated $\mathrm{NaHCO}_{3}$ solution. The aqueous phase was extracted with $\mathrm{CH}_{2} \mathrm{Cl}_{2}(3 \times 30 \mathrm{~mL})$ and the combined organic extracts were dried over anhydrous $\mathrm{Na}_{2} \mathrm{SO}_{4}$ and concentrated in vacuo. The resulting dark solid was purified by column chromatography (DCM:MeOH, 100:1, 100:5) yielding product 11 (49 mg, $85 \%)$ as a white amorphous solid.

$[\alpha]_{\mathrm{D}}^{20}=-11.2\left(c 0.25, \mathrm{CHCl}_{3}\right)$

${ }^{1}$ H NMR (500 MHz, Chloroform-d) $\delta 8.14(\mathrm{~d}, J=7.9 \mathrm{~Hz}, 1 \mathrm{H}), 7.23-7.27$ (m, 2H), 7.12-7.06 (m, 1H), 5.85-5.79 (m, 1H), 5.60-5.53 (m, 1H), 4.30-4.25 (m, 3H), $3.86(\mathrm{~s}$, $1 \mathrm{H}), 3.69(\mathrm{~d}, J=14.6 \mathrm{~Hz}, 1 \mathrm{H}), 3.53(\mathrm{~s}, 1 \mathrm{H}), 3.38-3.30(\mathrm{~m}, 1 \mathrm{H}), 3.12$ (dd, $J=17.4,6.6$ 
$\mathrm{Hz}, 1 \mathrm{H}), 3.06-2.97(\mathrm{~m}, 2 \mathrm{H}), 2.94-2.88(\mathrm{~m}, 1 \mathrm{H}), 2.32-2.28(\mathrm{~m}, 1 \mathrm{H}), 2.23-2.11(\mathrm{~m}, 2 \mathrm{H})$, $1.47(\mathrm{~d}, J=19.1 \mathrm{~Hz}, 1 \mathrm{H}), 0.90(\mathrm{~s}, 10 \mathrm{H}), 0.07$ (s, 6H).

${ }^{13}$ C NMR (126 MHz, Chloroform-d) $\delta$ 168.3 , 128.7 , 124.3 , $122.7,120.8,114.8$, $66.4,62.9,59.2,53.4,52.5,52.4,45.3,36.8,34.6,25.9,25.1,18.4,-5.1,-5.1$

HRMS (ESI) m/z: $[\mathrm{M}+\mathrm{H}]^{+}$Calcd for $\mathrm{C}_{27} \mathrm{H}_{37} \mathrm{~N}_{2} \mathrm{O}_{2} \mathrm{Si} 449.2619$; Found 449.2625.

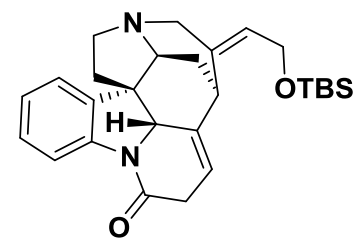

11

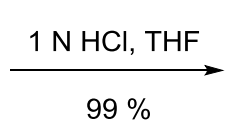

$99 \%$

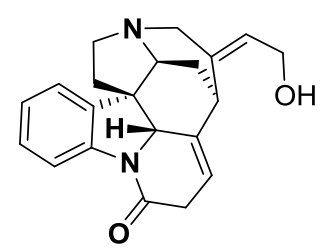

$(+)$-isostrychnine

A solution of silyl ether $11(20 \mathrm{mg}, 0.45 \mathrm{mmol})$ in $1 \mathrm{~N} \mathrm{HCl} \mathrm{(1} \mathrm{ml)} \mathrm{and} \mathrm{THF} \mathrm{(0.5}$ $\mathrm{ml}$ ) was stirred at room temperature for $3 \mathrm{~h}$. The mixture was then poured into satd. $\mathrm{NaHCO}_{3}$ and extracted with $\mathrm{CHCl}_{3}(3 \times 5 \mathrm{ml})$. The combined organics were dried over $\mathrm{NaSO}_{4}$ and concentrated in vacuo. The residue was submitted to flash chromatography on silica gel with $\mathrm{CH}_{2} \mathrm{Cl}_{2}-\mathrm{MeOH}$ (9:1)to afford (+)-isostrychnine (14.8 mg, 99\%)as white crystals ${ }^{[4]}$.

$[\alpha]_{\mathrm{D}}{ }^{20}=+17(c 0.2, \mathrm{EtOH})\left\{\right.$ lit. $^{[5]}[\alpha]_{\mathrm{D}}{ }^{20}=+23.7(c 0.31, \mathrm{EtOH})(99 \%$ ee $\left.)\right\}$

${ }^{1}$ H NMR (500 MHz, Chloroform-d) $\delta 8.15(\mathrm{~d}, J=8.1 \mathrm{~Hz}, 1 \mathrm{H}), 7.27-7.23(\mathrm{~m}, 1 \mathrm{H})$, $7.21-7.18(\mathrm{~m}, 1 \mathrm{H}), 7.09(\mathrm{td}, J=7.5,1.1 \mathrm{~Hz}, 1 \mathrm{H}), 5.87-5.84(\mathrm{~m}, 1 \mathrm{H}), 5.60(\mathrm{t}, J=6.7$ $\mathrm{Hz}, 1 \mathrm{H}), 4.32-4.24(\mathrm{~m}, 3 \mathrm{H}), 3.70(\mathrm{~s}, 1 \mathrm{H}), 3.65-3.54(\mathrm{~m}, 2 \mathrm{H}), 3.28-3.19(\mathrm{~m}, 1 \mathrm{H})$, $3.12(\mathrm{dd}, J=17.4,6.7 \mathrm{~Hz}, 1 \mathrm{H}), 3.04-2.99(\mathrm{~m}, 1 \mathrm{H}), 2.96(\mathrm{~d}, J=14.4 \mathrm{~Hz}, 1 \mathrm{H}), 2.90(\mathrm{dt}$, $J=11.1,7.2 \mathrm{~Hz}, 1 \mathrm{H}), 2.29(\mathrm{dt}, J=13.3,7.8 \mathrm{~Hz}, 1 \mathrm{H}), 2.21(\mathrm{dd}, J=7.0,5.2 \mathrm{~Hz}, 1 \mathrm{H})$,

2.18-2.13 (m, 2H), $1.48(\mathrm{dt}, J=14.1,2.5 \mathrm{~Hz}, 1 \mathrm{H})$.

${ }^{13}$ C NMR (126 MHz, Chloroform-d) $\delta 168.5,142.5$,141.6, 134.7, 128.6 , 126.6 , $124.3,122.7,120.8,114.8,67.4,63.5$, 58.5 , 54.1 , 53.0 , 52.6 , 46.3 , 37.1 , 34.9 , 25.9 .

HRMS (ESI) m/z: [M + H] ${ }^{+}$Calcd for $\mathrm{C}_{21} \mathrm{H}_{23} \mathrm{~N}_{2} \mathrm{O}_{2}$ 335.1754; Found 335.1756. 


\section{The comparison of the spectra data}

Table S1 the comparison of ${ }^{1} \mathrm{H}$ NMR data

\begin{tabular}{|c|c|c|c|c|c|c|}
\hline \multicolumn{3}{|c|}{$\begin{array}{c}\text { Synthetic Isostrychnine } \\
\left.{ }^{1} \mathrm{H} \text { NMR(500 MHz, } \mathrm{CDCl}_{3}\right)\end{array}$} & \multicolumn{3}{|c|}{$\begin{array}{c}\text { Reported }^{[6]} \\
\left.{ }^{1} \mathrm{H} \text { NMR(500 MHz, } \mathrm{CDCl}_{3}\right)\end{array}$} & \multirow{2}{*}{$\begin{array}{c}\text { Deviation } \\
\Delta \delta(\mathrm{ppm})\end{array}$} \\
\hline$\delta(\mathrm{ppm})$ & Integration & Multiplicity & $\delta(\mathrm{ppm})$ & Integration & Multiplicity & \\
\hline 8.15 & $1 \mathrm{H}$ & $\mathrm{d}(J=8.1 \mathrm{~Hz})$ & 8.15 & $1 \mathrm{H}$ & $\mathrm{d}(J=8.0 \mathrm{~Hz})$ & 0 \\
\hline $7.27-7.23$ & $1 \mathrm{H}$ & $\mathrm{m}$ & $7.26-7.23$ & $1 \mathrm{H}$ & $\mathrm{m}$ & 0 \\
\hline $7.21-7.18$ & $1 \mathrm{H}$ & $\mathrm{m}$ & 7.21-7.18 & $1 \mathrm{H}$ & $\mathrm{m}$ & 0 \\
\hline 7.09 & $1 \mathrm{H}$ & $\operatorname{Td}(J=7.5,1.1 \mathrm{~Hz})$ & 7.09 & $1 \mathrm{H}$ & $\operatorname{td}(J=7.5,1.0 \mathrm{~Hz})$ & 0 \\
\hline $5.87-5.84$ & $1 \mathrm{H}$ & $\mathrm{m}$ & $5.87-5.84$ & $1 \mathrm{H}$ & $\mathrm{m}$ & 0 \\
\hline 5.60 & $1 \mathrm{H}$ & $\mathrm{t}(J=6.7 \mathrm{~Hz})$ & 5.59 & $1 \mathrm{H}$ & $\mathrm{t}(J=6.5 \mathrm{~Hz})$ & 0.01 \\
\hline $4.32-4.24$ & $3 \mathrm{H}$ & $\mathrm{m}$ & $4.31-4.24$ & $3 \mathrm{H}$ & $\mathrm{m}$ & 0 \\
\hline 3.70 & $1 \mathrm{H}$ & $\mathrm{s}$ & $3.68-3.66$ & $1 \mathrm{H}$ & $\mathrm{m}$ & 0.03 \\
\hline $3.65-3.57$ & $2 \mathrm{H}$ & $\mathrm{m}$ & $3.61-3.58$ & $2 \mathrm{H}$ & $\mathrm{m}$ & 0.01 \\
\hline $3.28-3.19$ & $1 \mathrm{H}$ & $\mathrm{m}$ & 3.21 & $1 \mathrm{H}$ & $\begin{array}{c}\operatorname{ddd}(J=11.1,7.8 \\
5.3 \mathrm{~Hz})\end{array}$ & 0.03 \\
\hline 3.12 & $1 \mathrm{H}$ & $\mathrm{dd}(J=17.4,6.7 \mathrm{~Hz})$ & 3.12 & $1 \mathrm{H}$ & $\mathrm{dd}(J=17.4,6.7 \mathrm{~Hz})$ & 0 \\
\hline $3.04-2.99$ & $1 \mathrm{H}$ & $\mathrm{m}$ & $3.05-2.98$ & $1 \mathrm{H}$ & $\mathrm{m}$ & 0 \\
\hline 2.96 & $1 \mathrm{H}$ & $\mathrm{d}(J=14.4 \mathrm{~Hz})$ & 2.94 & $1 \mathrm{H}$ & $\mathrm{d}(J=14.4 \mathrm{~Hz})$ & 0.02 \\
\hline 2.90 & $1 \mathrm{H}$ & $\mathrm{dt}(J=11.1,7.2 \mathrm{~Hz})$ & 2.89 & $1 \mathrm{H}$ & $\mathrm{dt}(J=11.1,7.1 \mathrm{~Hz})$ & 0.01 \\
\hline 2.29 & $1 \mathrm{H}$ & $\mathrm{dt}(J=13.3,7.8 \mathrm{~Hz})$ & 2.28 & $1 \mathrm{H}$ & $\mathrm{dt}(J=13.1,7.7 \mathrm{~Hz})$ & 0.01 \\
\hline 2.21 & $1 \mathrm{H}$ & $\mathrm{dd}(J=7.0,5.2 \mathrm{~Hz})$ & 2.21 & $1 \mathrm{H}$ & $\mathrm{dd}(J=13.1,6.1 \mathrm{~Hz})$ & 0 \\
\hline $2.18-2.13$ & $2 \mathrm{H}$ & $\mathrm{m}$ & $2.18-2.12$ & $1 \mathrm{H}$ & $\mathrm{m}$ & 0 \\
\hline 1.48 & $1 \mathrm{H}$ & $\mathrm{dt}(J=14.1,2.5 \mathrm{~Hz})$ & 1.47 & $1 \mathrm{H}$ & $\mathrm{dt}(J=14.0,2.3 \mathrm{~Hz})$ & 0.01 \\
\hline
\end{tabular}


Table S2 The comparison of ${ }^{13} \mathrm{C}$ NMR data

\begin{tabular}{|c|c|c|}
\hline $\begin{array}{l}\text { Synthetic Isostrychnine } \\
\qquad \begin{array}{c}\left(126 \mathrm{MHz}, \mathrm{CDCl}_{3}\right) \\
\delta(\mathrm{ppm})\end{array}\end{array}$ & $\begin{array}{c}\text { Reported }^{[6]} \\
\left(125 \mathrm{MHz}, \mathrm{CDCl}_{3}\right) \\
\delta(\mathrm{ppm})\end{array}$ & $\begin{array}{l}\text { Deviation } \\
\Delta \delta(\mathrm{ppm})\end{array}$ \\
\hline 168.5 & 168.5 & 0 \\
\hline 142.5 & 142.1 & 0.4 \\
\hline 141.6 & 141.3 & 0.3 \\
\hline- & 137.1 & - \\
\hline 134.7 & 134.5 & 0.2 \\
\hline 128.6 & 128.4 & 0.2 \\
\hline 126.6 & 126.7 & -0.1 \\
\hline 124.3 & 124.2 & 0.1 \\
\hline 122.7 & 122.5 & 0.2 \\
\hline 120.8 & 120.5 & 0.3 \\
\hline 114.8 & 114.6 & 0.2 \\
\hline 67.4 & 67.2 & 0.2 \\
\hline 63.5 & 63.3 & 0.2 \\
\hline 58.5 & 58.1 & 0.4 \\
\hline 54.1 & 53.9 & 0.2 \\
\hline 53.0 & 52.8 & 0.2 \\
\hline 52.6 & 52.2 & 0.4 \\
\hline 46.3 & 46.1 & 0.2 \\
\hline 37.1 & 36.8 & 0.3 \\
\hline 34.9 & 34.6 & 0.3 \\
\hline 25.9 & 25.7 & 0.2 \\
\hline
\end{tabular}




\section{References}

[1] He, W., Hu, J., Wang, P., Le, C.,Chen, L., Ji, K., Yang, S., Li, Y., Xie, Z., Xie, W. Highly enantioselective tandem michael addition of tryptamine-derived oxindoles to alkynones: concise synthesis of strychnos alkaloids. Angew. Chem. Int. Ed. 2018, 57, 3806 -3809 .

[2] Qi, W., Mcintosh, M. C. Acyclic 1,4-stereocontrol via reductive 1,3-transpositions. Org. Lett. 2008, 10, 357-359.

[3] Beemelmanns, D., Reissig H.-U. A short formal total synthesis of strychnine with a samarium diiodide induced cascade reaction as the key step. Angew. Chem. Int. Ed. 2010, $49,8021-8025$.

[4] Rawal, V. H., Iwasa, S. A short stereocontrolled synthesis of strychnine. J. Org. Chem. 1994, 59, 10, 2685-2686.

[5] Mori, M., Nakanishi, M., Kajishima, D., Sato, Y. A novel and general synthetic pathway to strychnos indole alkaloids: total syntheses of (-)-tubifoline, (-)-dehydrotubifoline, and (-)-strychnine using palladium-catalyzed asymmetric allylic substitution. J. Am. Chem. Soc. 2003, 125, 32, 9801-9807.

[6] Zhao, S., Teijaro, C. N., Chen, H., Sirasani, G., Andrade, R. B. Synthesis of bis-strychnos alkaloids (-)-sungucine, (-)-isosungucine, and (-)-strychnogucine B from (-)-strychnine. $J$. Braz. Chem.l Soc. 2019, 30, 436-453. 


\section{HPLC date}<smiles>COC(=O)CCCC(=O)CC1N([18OH])CCC12C(=O)Nc1ccccc12</smiles>

HPLC Report

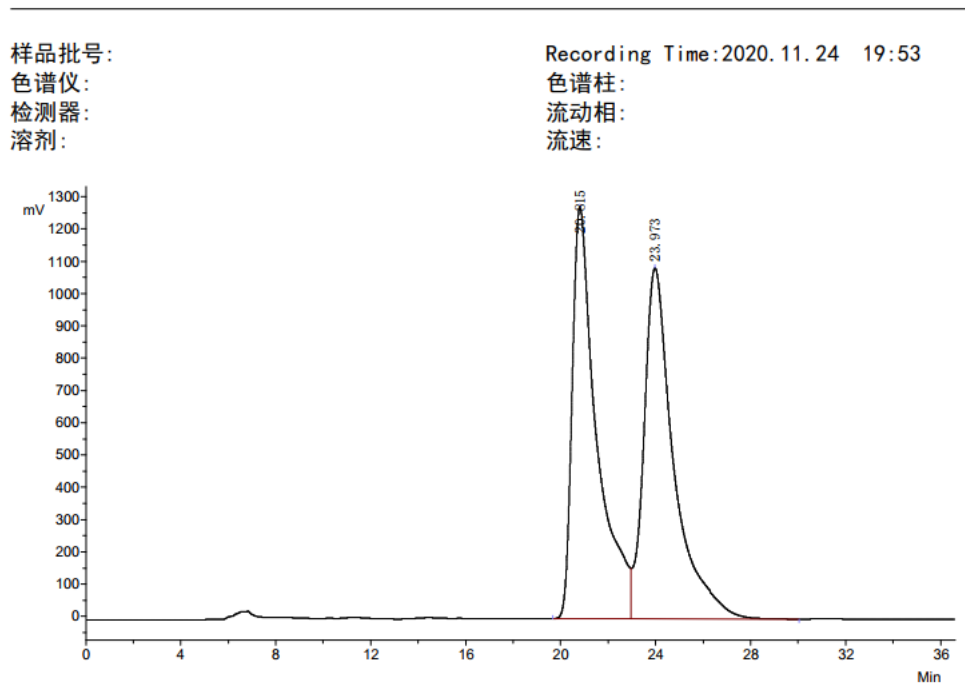

\begin{tabular}{rrrrrl} 
No. & R. Time & PeakHeight & PeakArea & PerCent & \multicolumn{1}{l}{ Conc } \\
\hline 1 & 20.815 & 1273607.4 & 91645946.4 & 49.1195 & 49.1195 \\
2 & 23.973 & 1086849.2 & 94931708.5 & 50.8805 & 50.8805 \\
\hline Total & 2360456.6 & \multirow{2}{*}{186577654.9} & 100.0000 & 100.0000
\end{tabular}

样品批号： Recording Time:2020.11.24 20:42

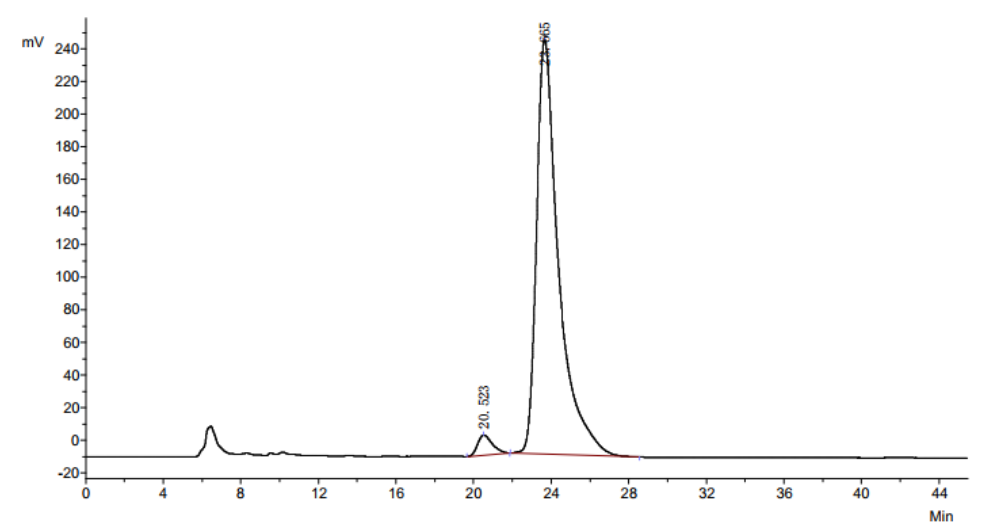

\begin{tabular}{rrrrrr} 
No. & R. Time & PeakHeight & \multicolumn{1}{c}{ PeakArea } & PerCent & \multicolumn{1}{c}{ Conc } \\
\hline 1 & 20.523 & 12344.7 & 650922.3 & 3.1271 & 3.1271 \\
2 & 23.665 & 254326.6 & 20164823.2 & 96.8729 & 96.8729 \\
\hline Total & 266671.2 & 20815745.5 & 100.0000 & 100.0000
\end{tabular}




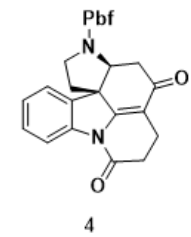

HPLC Report

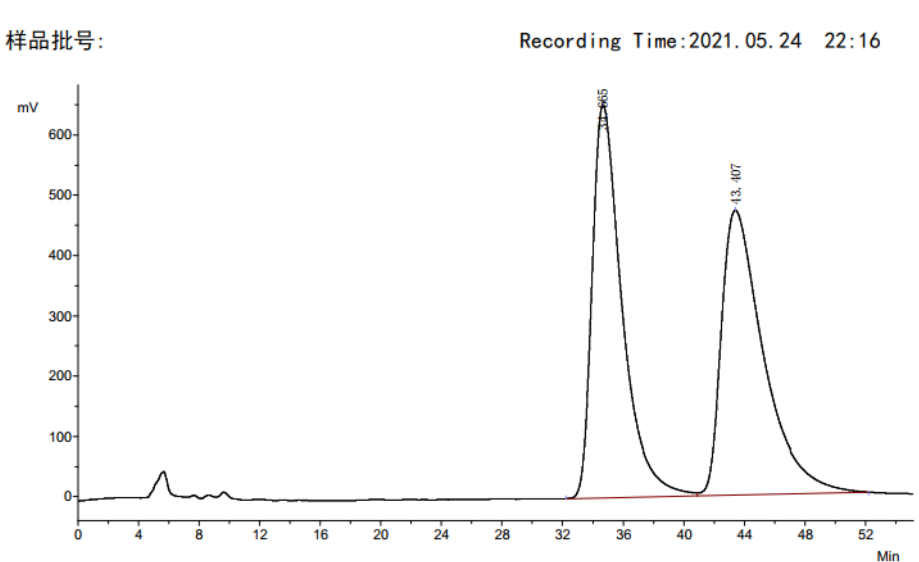

\begin{tabular}{rrrrrl} 
No. & R. Time & PeakHeight & PeakArea & PerCent & \multicolumn{1}{l}{ Conc } \\
\hline 1 & 34.665 & 651582.6 & 89205567.8 & 49.4036 & 49.4036 \\
2 & 43.407 & 472421.3 & 91359485.8 & 50.5964 & 50.5964 \\
\hline Total & 1124003.9 & 180565053.6 & 100.0000 & 100.0000
\end{tabular}

HPLC Report

样品批号:

$\mathrm{mv}$

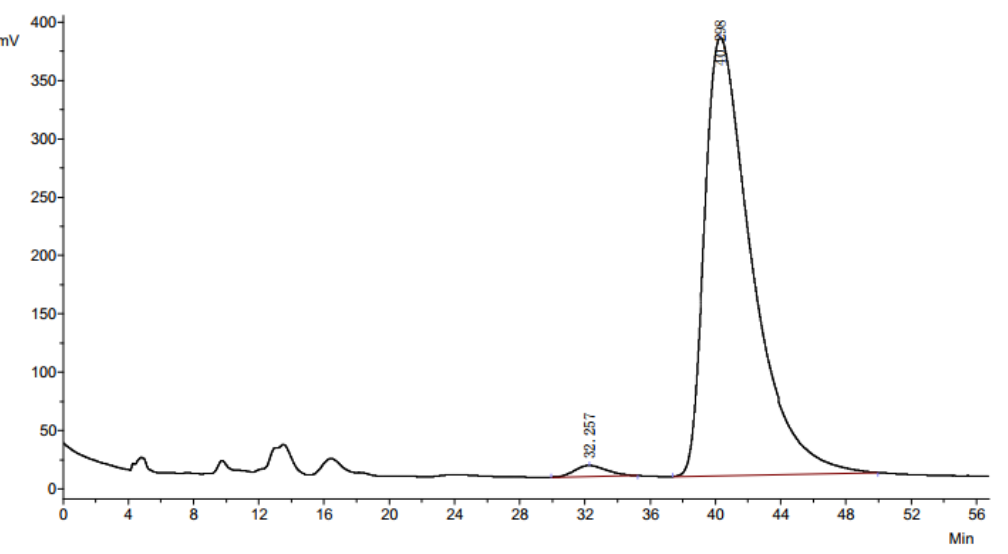

\begin{tabular}{rrrrrr} 
No. & R. Time & PeakHeight & \multicolumn{1}{c}{ PeakArea } & PerCent & \multicolumn{1}{c}{ Conc } \\
\hline 1 & 32.257 & 9506.7 & 1291722.7 & 1.6863 & 1.6863 \\
2 & 40.298 & 375253.5 & 75310945.0 & 98.3137 & 98.3137 \\
\hline Total & 384760.3 & 76602667.7 & 100.0000 & 100.0000
\end{tabular}




\section{NMR spectra}

${ }^{1} \mathrm{H}$ NMR Spectrum of $N_{\mathrm{b}}$-Pbf-tryptamine (500 MHz, CDCl $)$

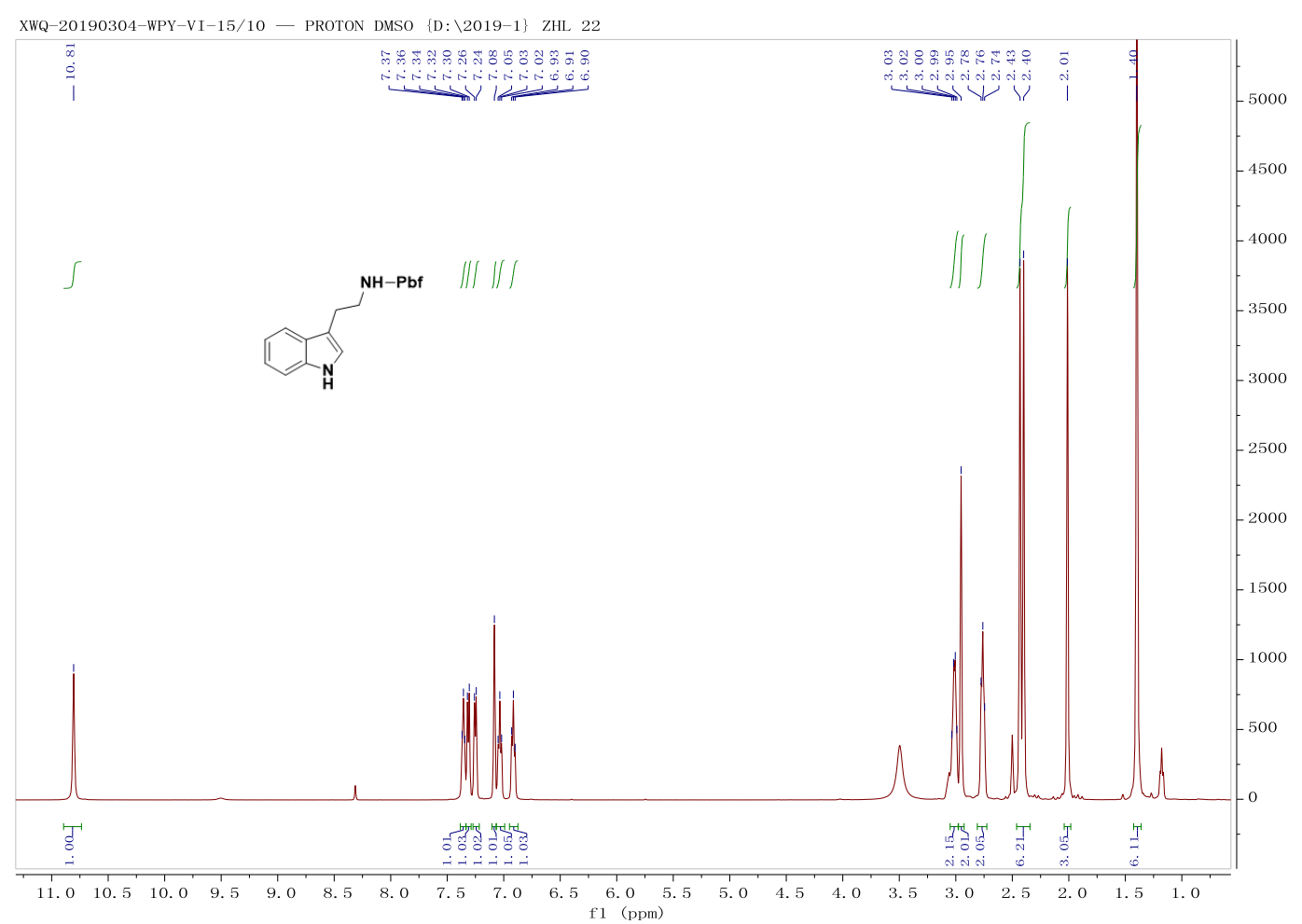

${ }^{13} \mathrm{C}$ NMR Spectrum of $N_{\mathrm{b}}$-Pbf-tryptamine (126 MHz, $\left.\mathrm{CDCl}_{3}\right)$

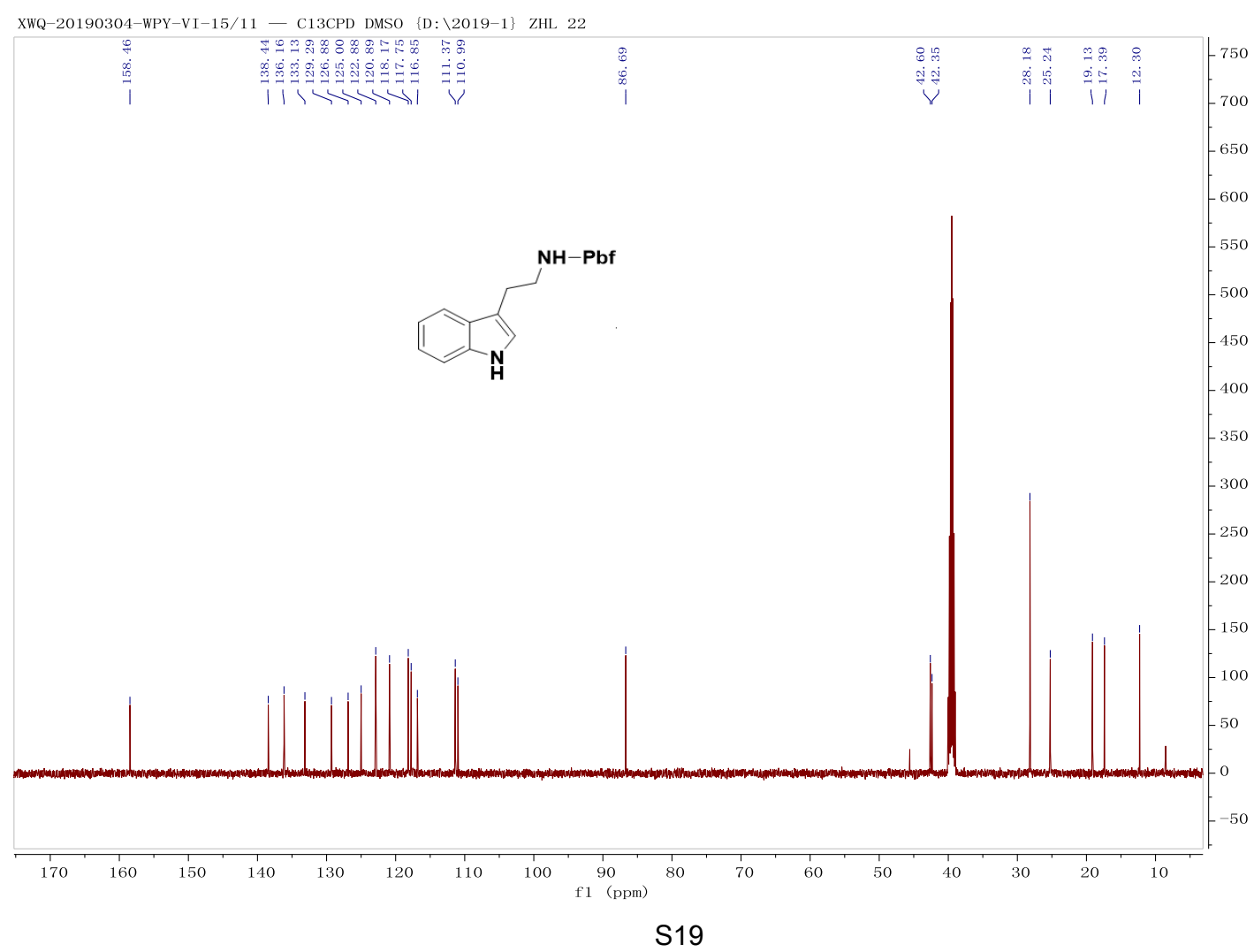




\section{${ }^{1} \mathrm{H}$ NMR Spectrum of $6\left(500 \mathrm{MHz}, \mathrm{CDCl}_{3}\right)$}

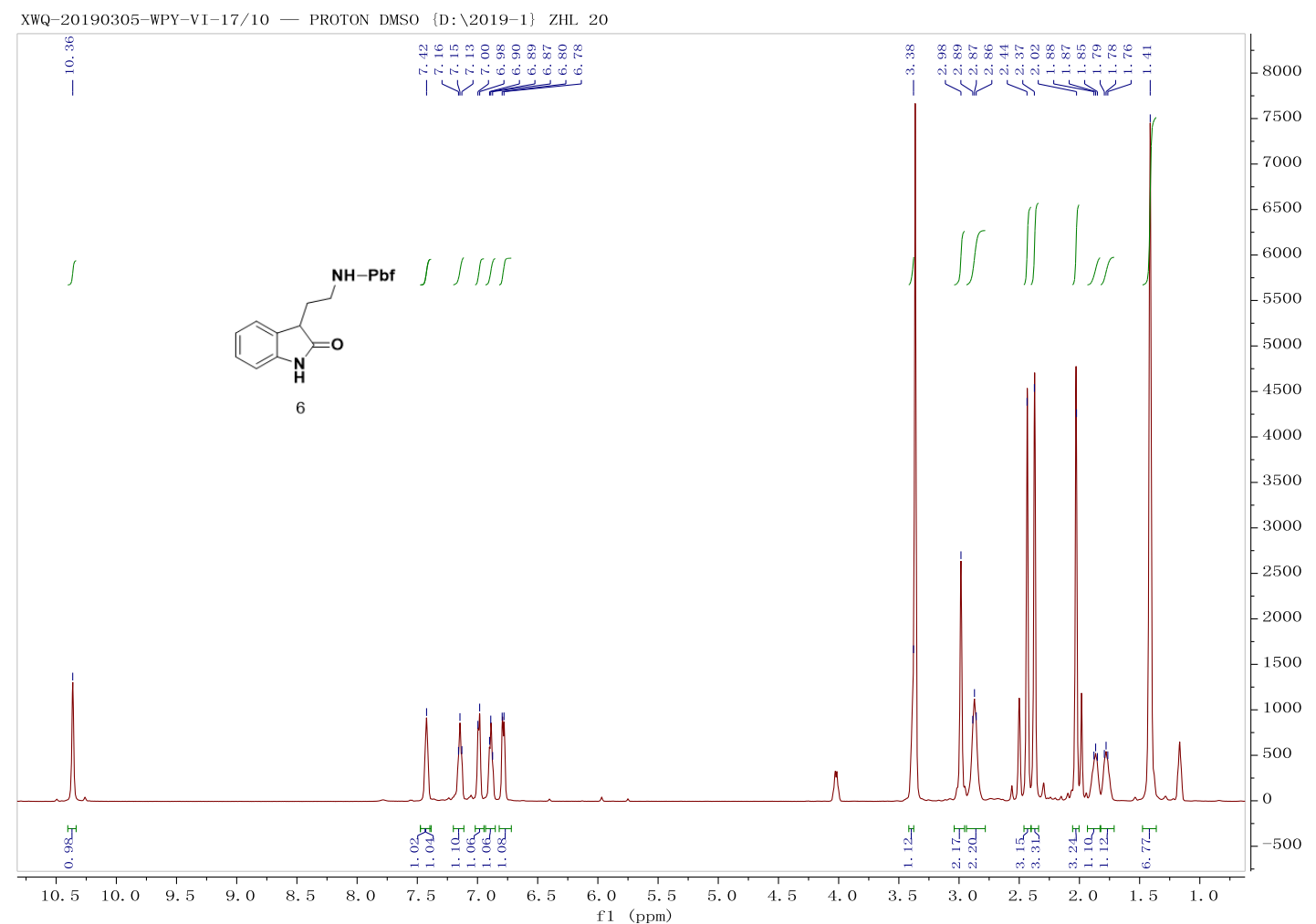

${ }^{13} \mathrm{C}$ NMR Spectrum of $6\left(126 \mathrm{MHz}, \mathrm{CDCl}_{3}\right)$

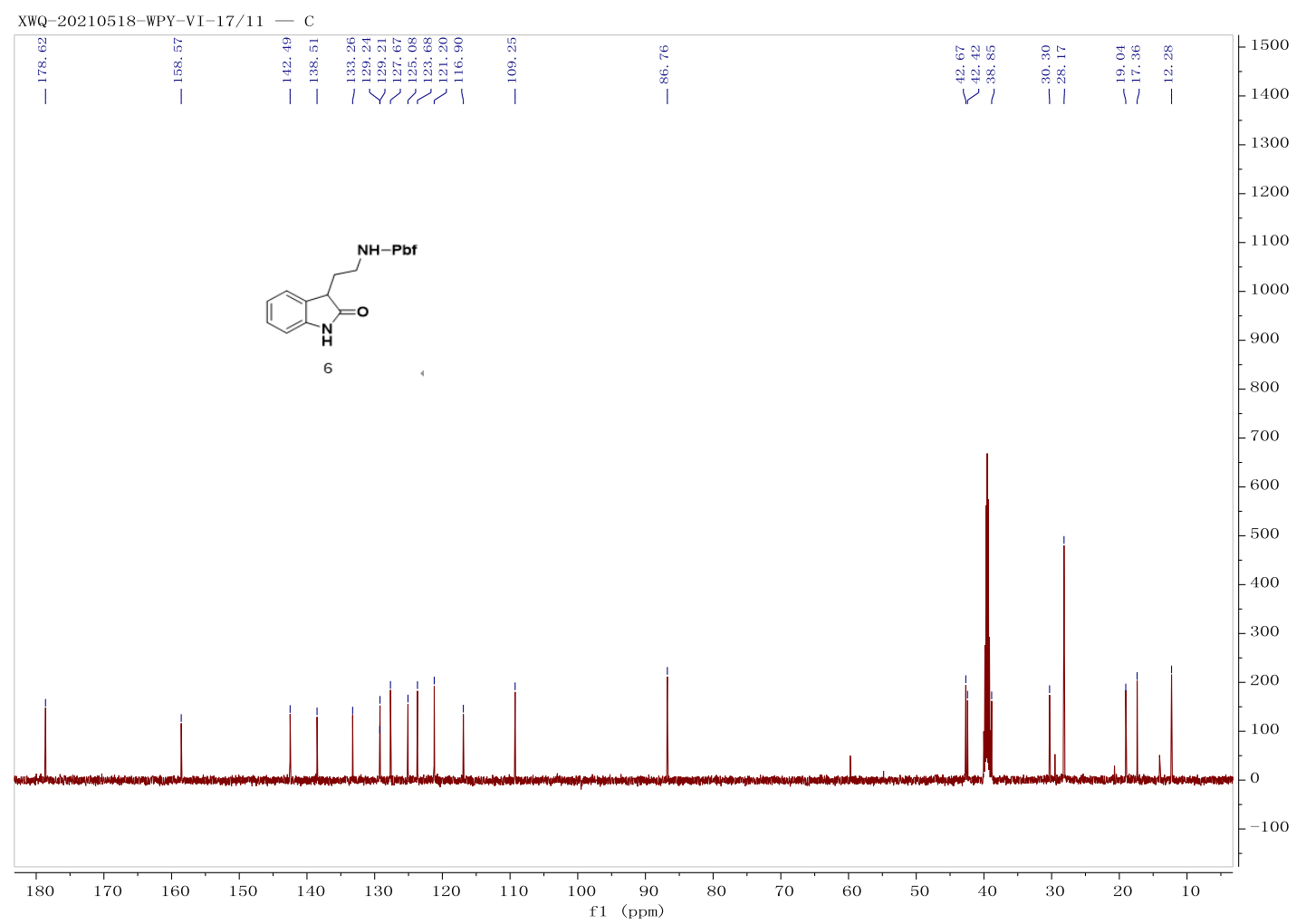




\section{${ }^{1} \mathrm{H}$ NMR Spectrum of $5\left(500 \mathrm{MHz}, \mathrm{CDCl}_{3}\right)$}

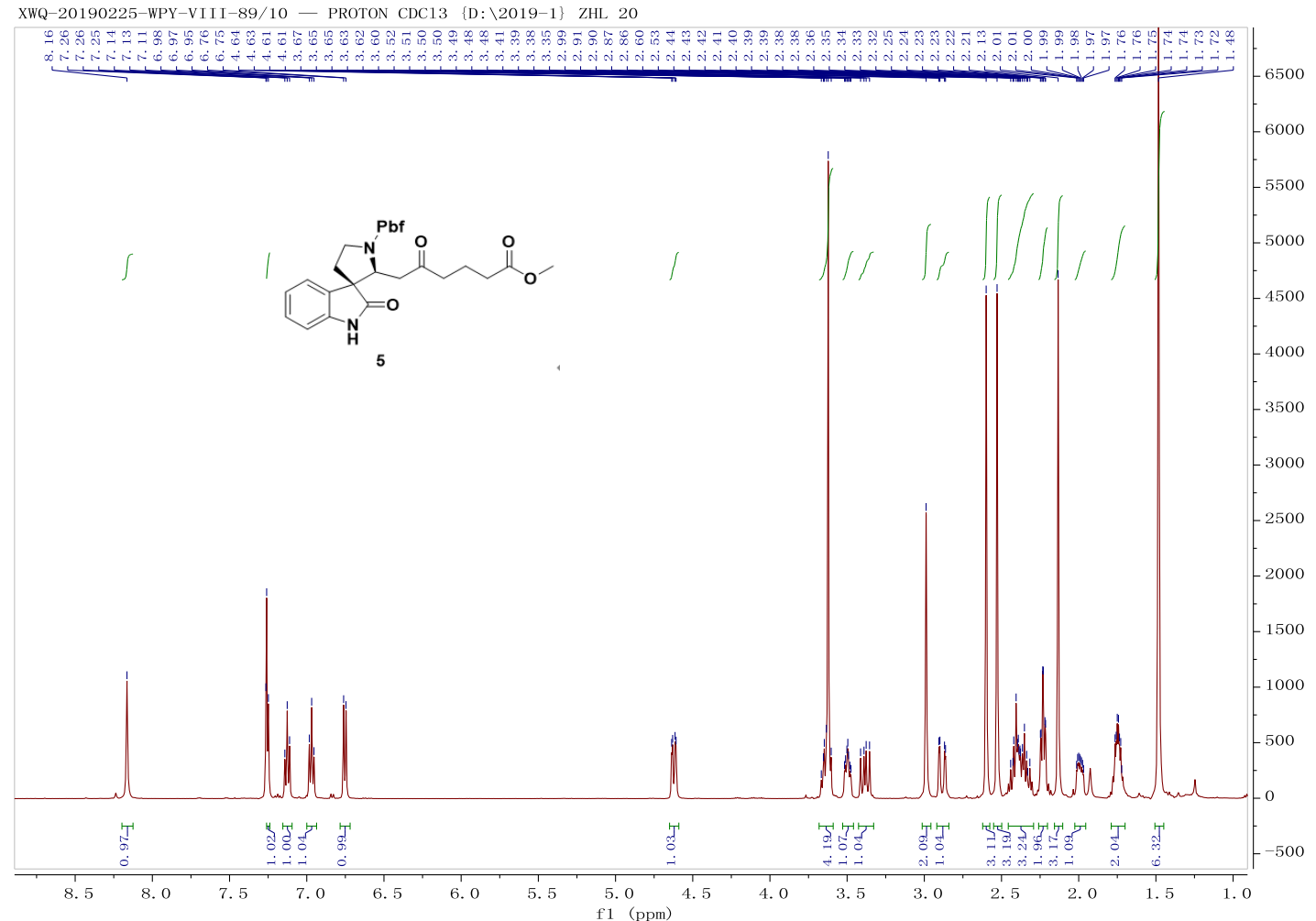

${ }^{13} \mathrm{C}$ NMR Spectrum of $5\left(126 \mathrm{MHz}, \mathrm{CDCl}_{3}\right)$

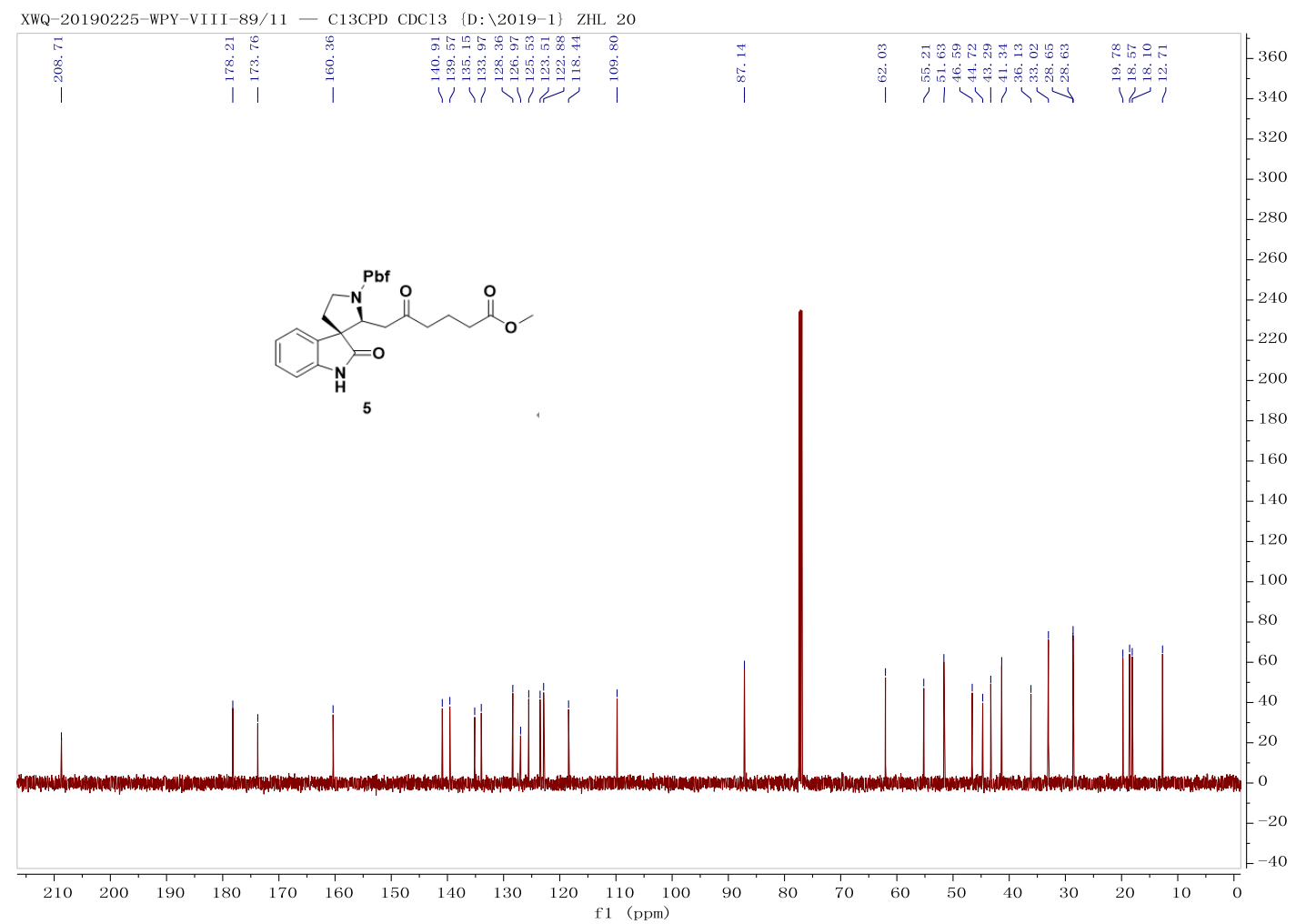




\section{${ }^{1} \mathrm{H}$ NMR Spectrum of $5^{\prime}\left(500 \mathrm{MHz}, \mathrm{CDCl}_{3}\right)$}

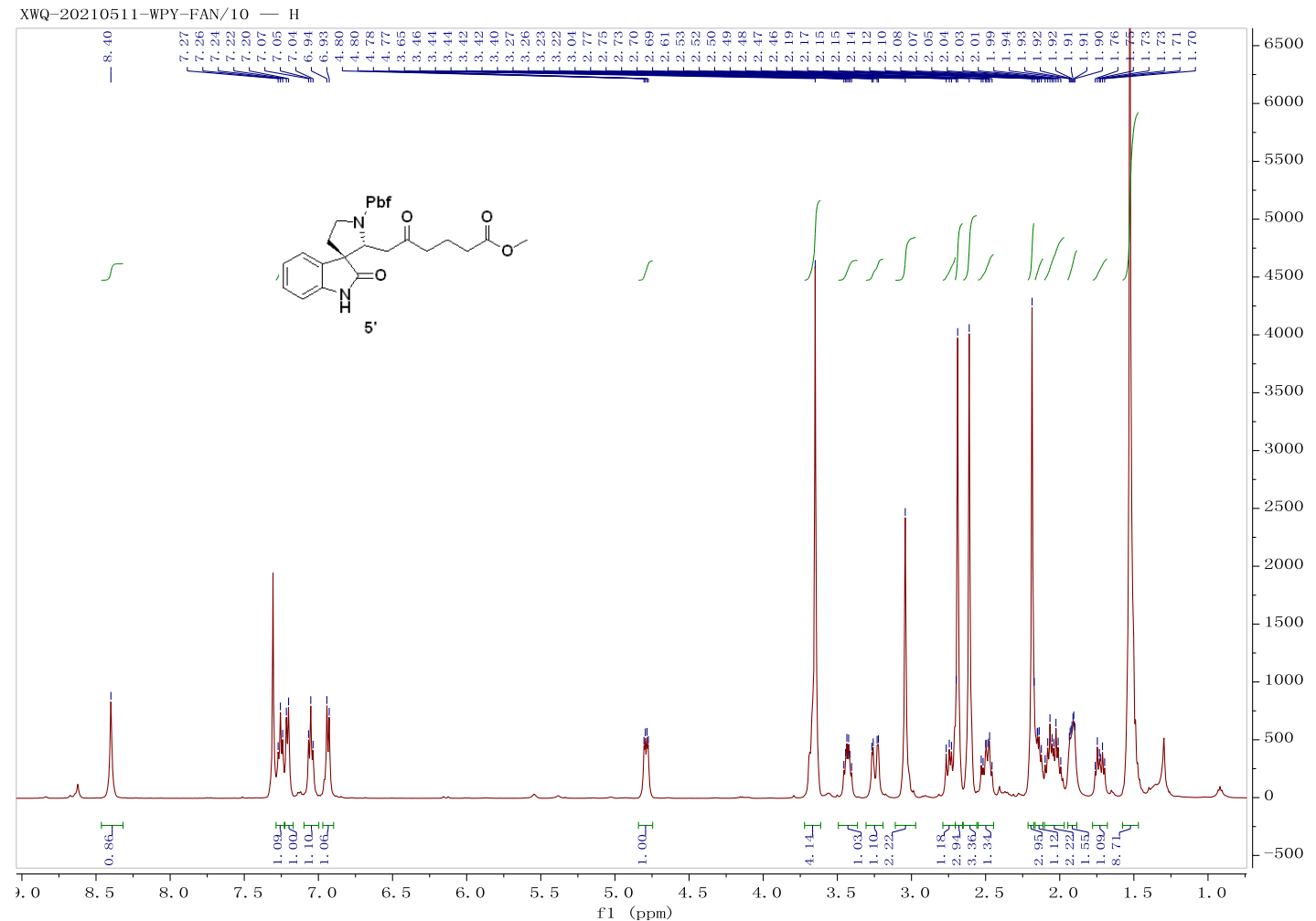

${ }^{13} \mathrm{C}$ NMR Spectrum of 5' (126 MHz, $\left.\mathrm{CDCl}_{3}\right)$

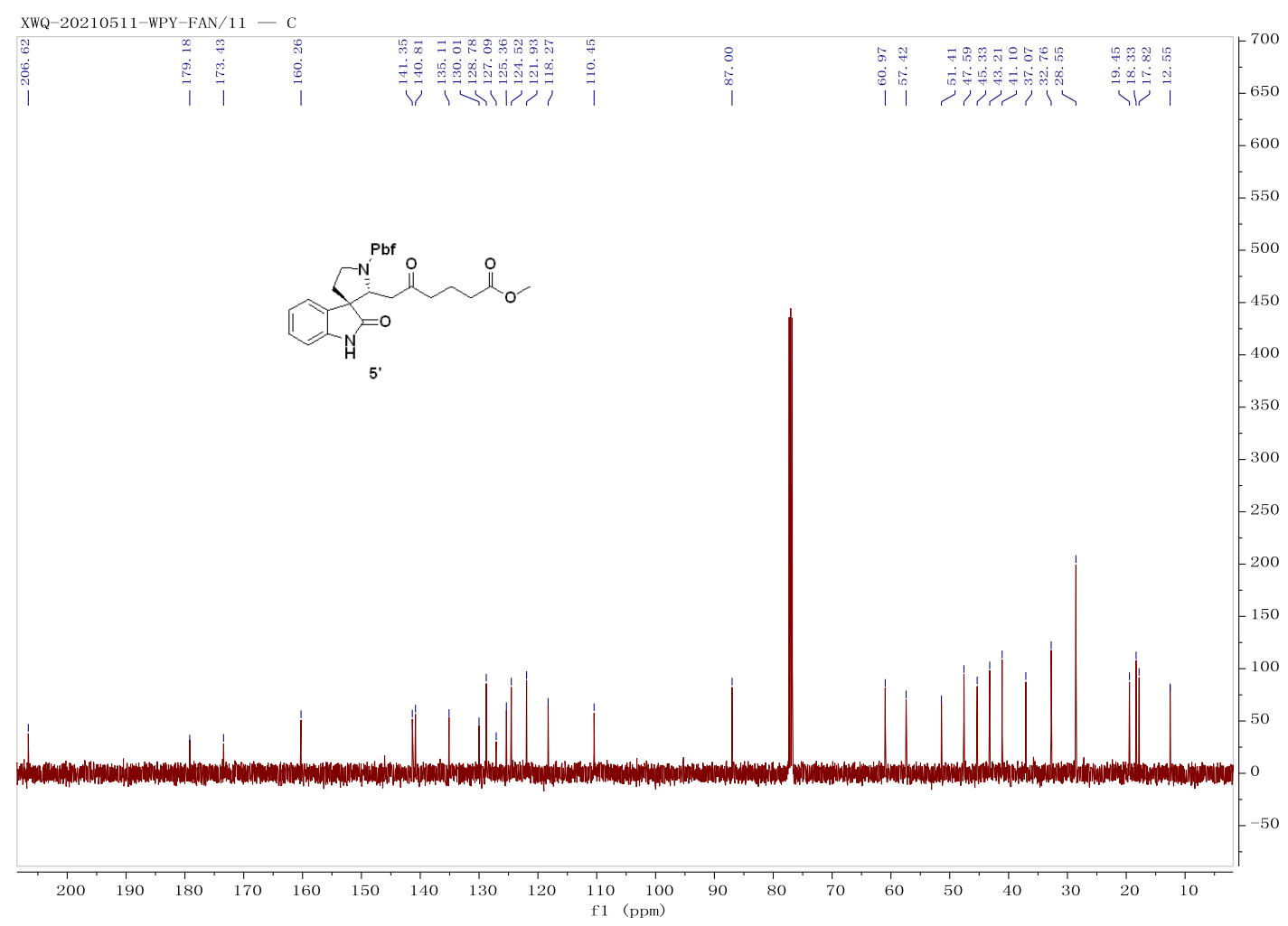




\section{${ }^{1} \mathrm{H}$ NMR Spectrum of $8\left(500 \mathrm{MHz}, \mathrm{CDCl}_{3}\right)$}

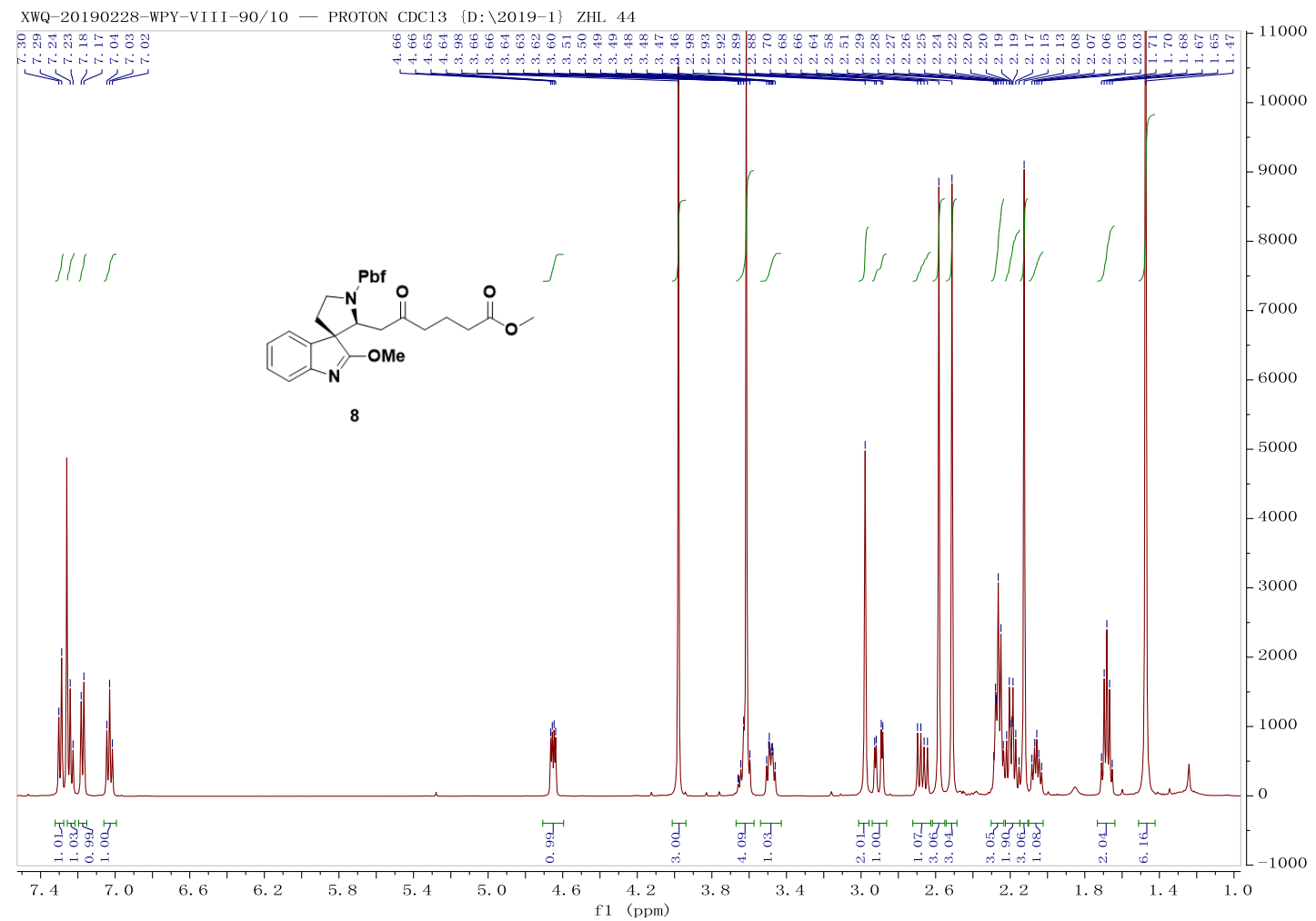

${ }^{13} \mathrm{C}$ NMR Spectrum of $8\left(126 \mathrm{MHz}, \mathrm{CDCl}_{3}\right)$

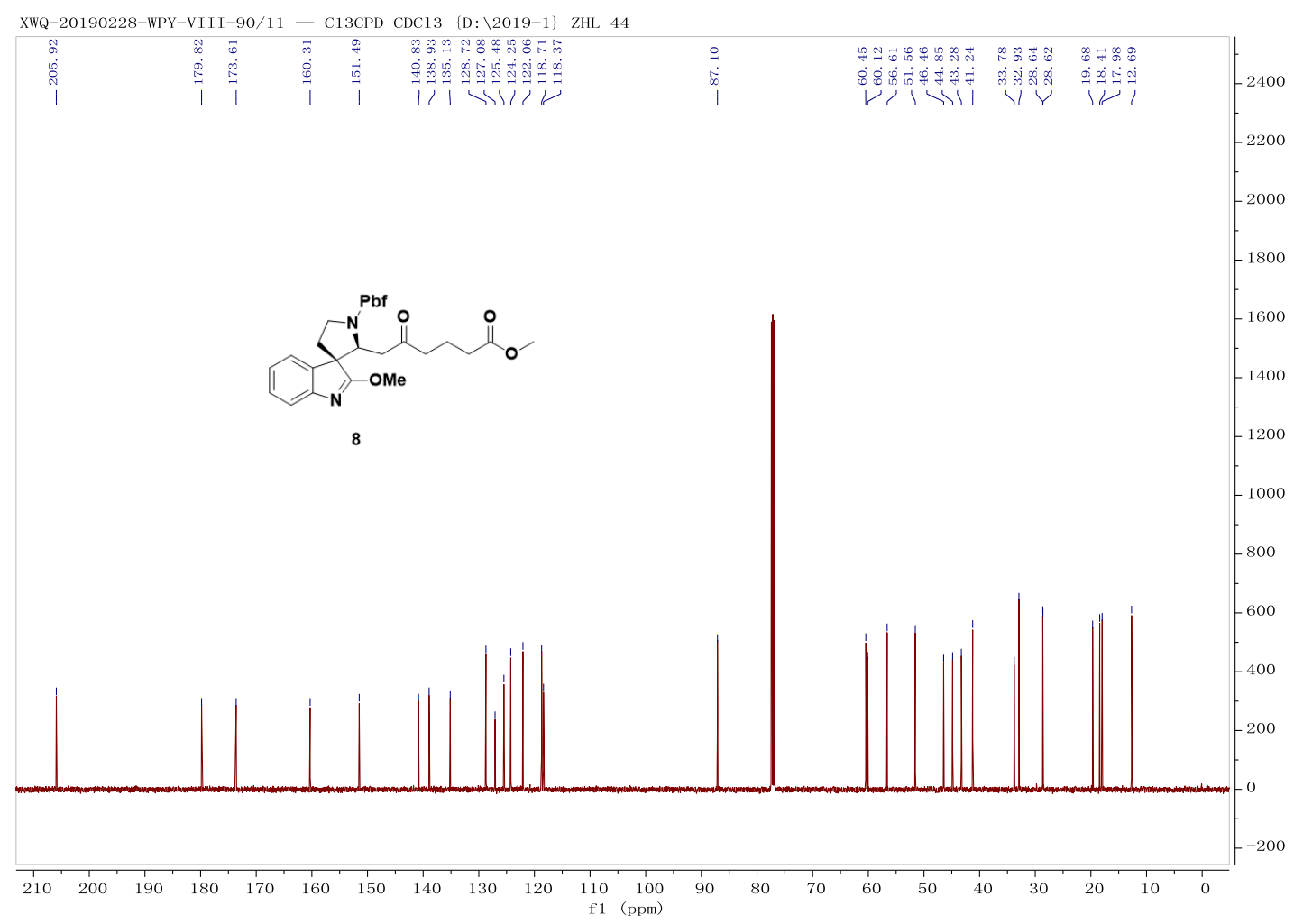




\section{${ }^{1} \mathrm{H}$ NMR Spectrum of $4\left(500 \mathrm{MHz}, \mathrm{CDCl}_{3}\right)$}

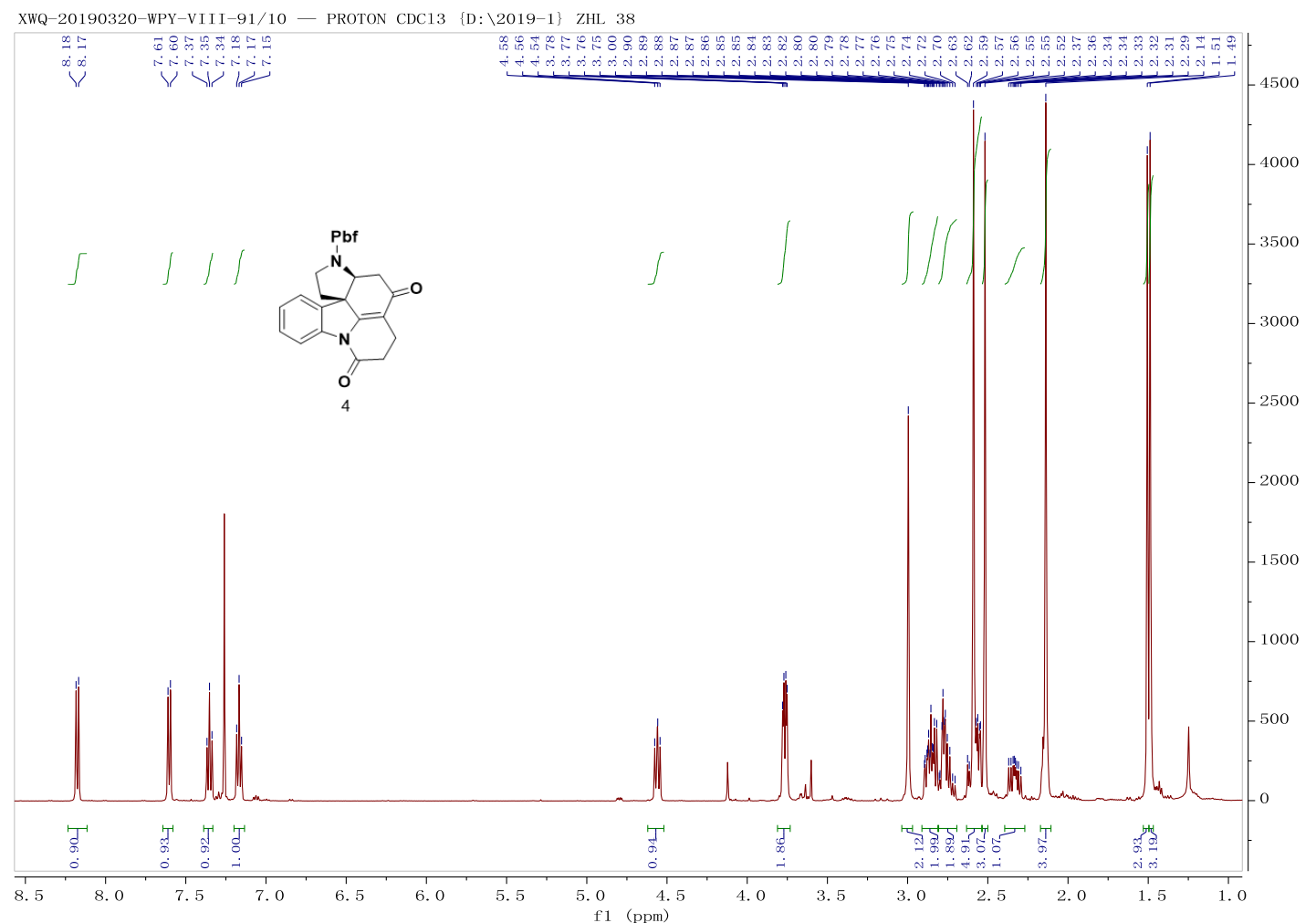

${ }^{13} \mathrm{C}$ NMR Spectrum of $4\left(126 \mathrm{MHz}, \mathrm{CDCl}_{3}\right)$

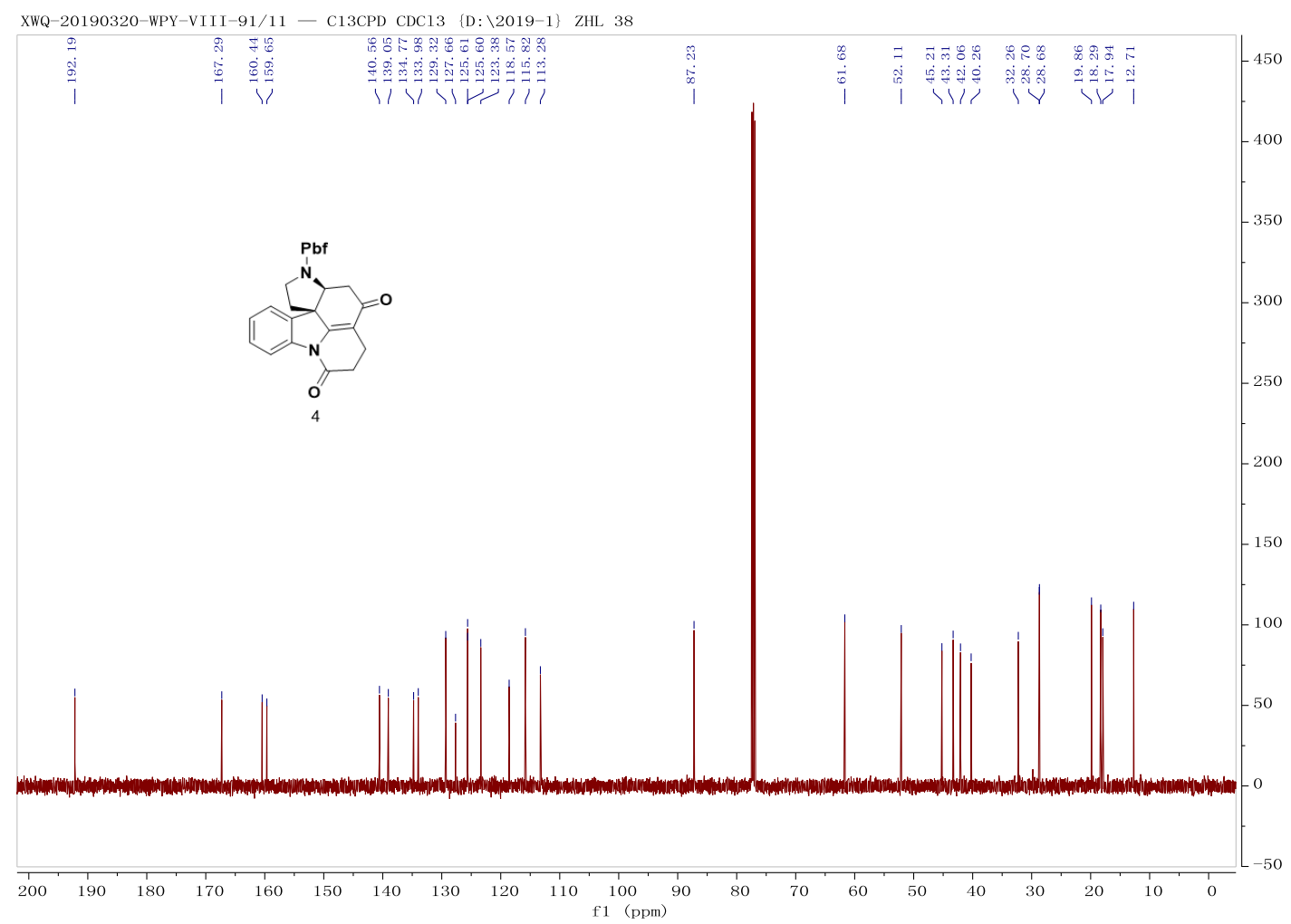




\section{${ }^{1} \mathrm{H}$ NMR Spectrum of $4 \mathrm{a}\left(500 \mathrm{MHz}, \mathrm{CDCl}_{3}\right)$}

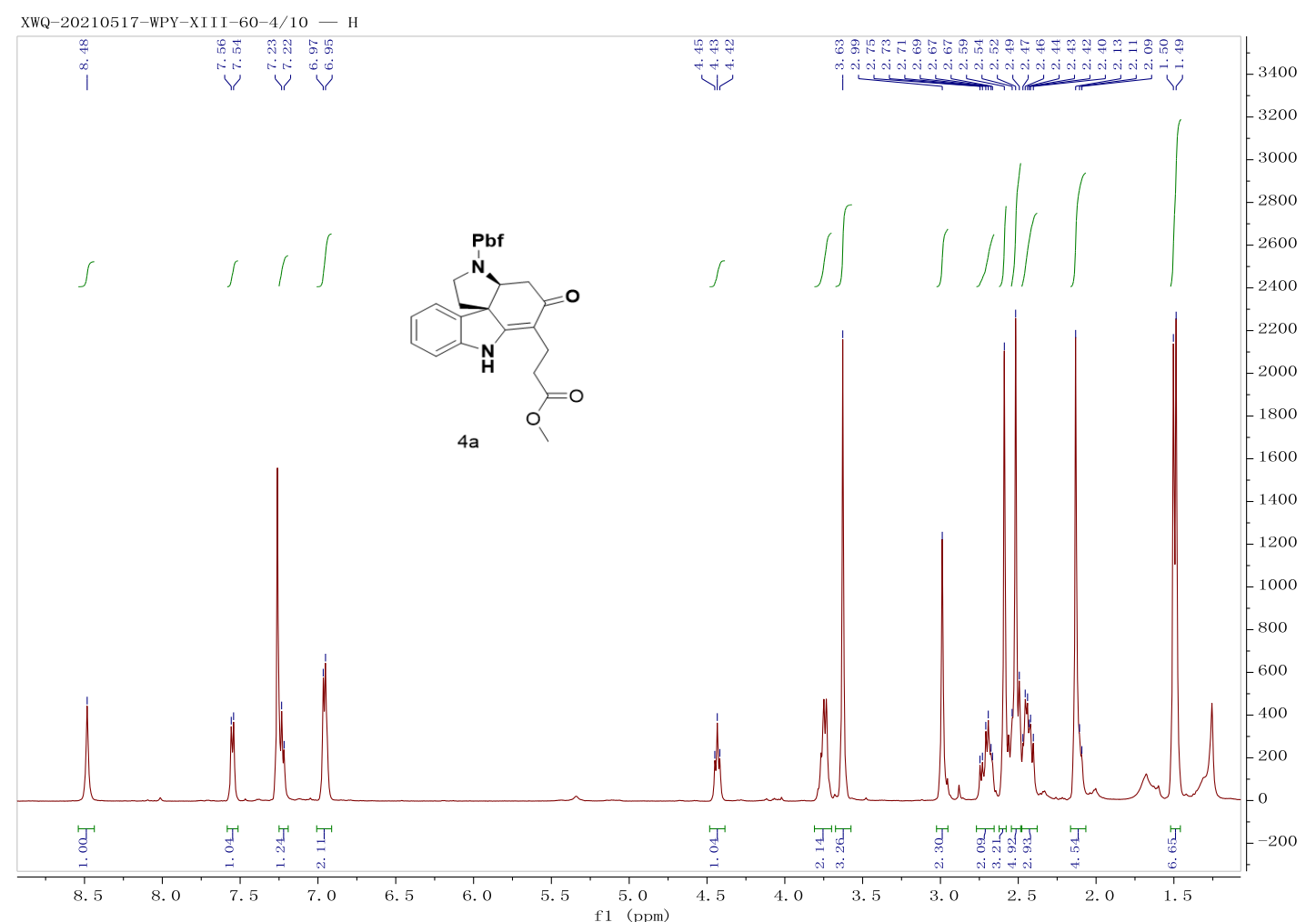

${ }^{13} \mathrm{C}$ NMR Spectrum of $4 \mathrm{a}\left(126 \mathrm{MHz}, \mathrm{CDCl}_{3}\right)$

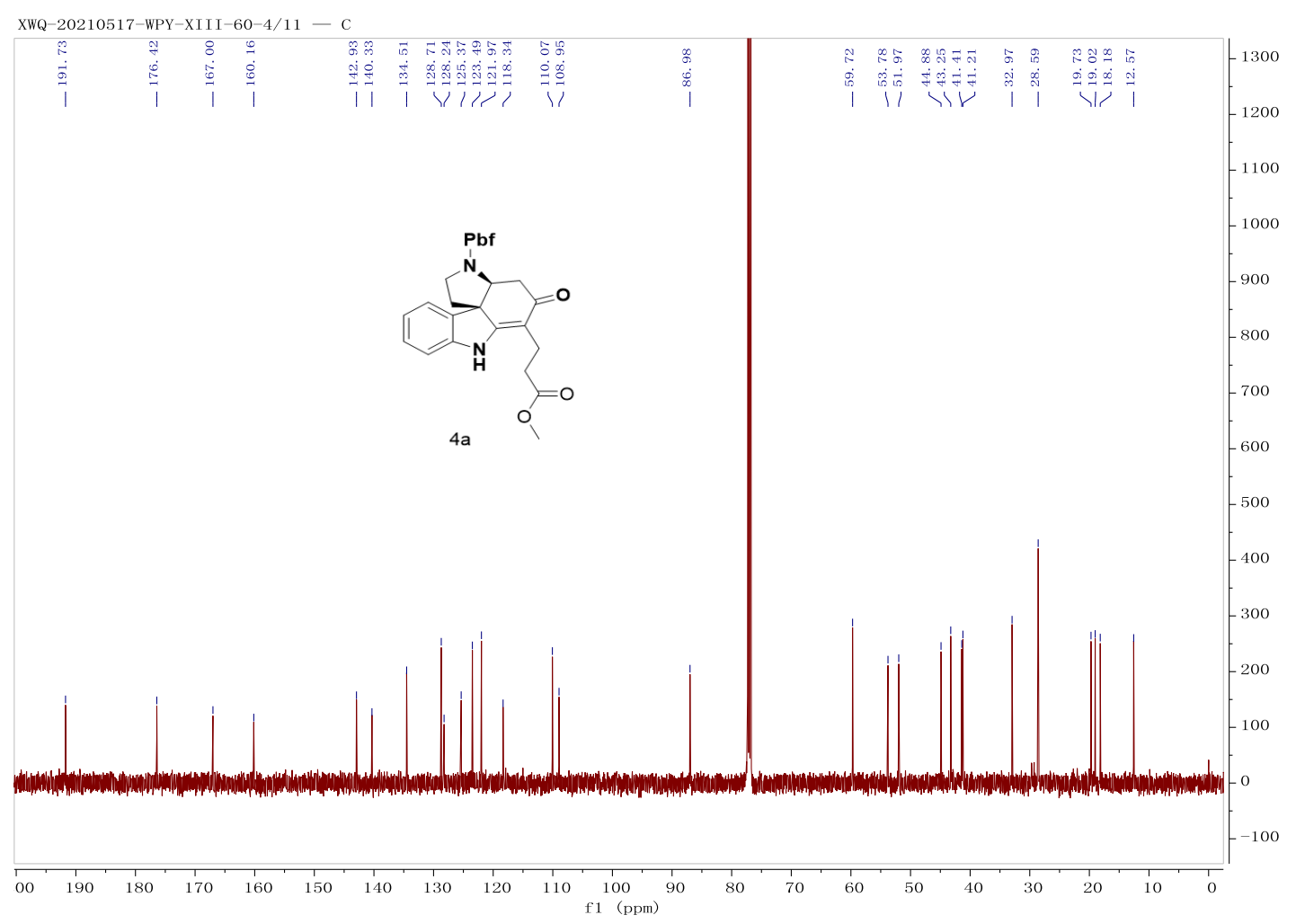




\section{${ }^{1} \mathrm{H}$ NMR Spectrum of $9\left(500 \mathrm{MHz}, \mathrm{CDCl}_{3}\right)$}

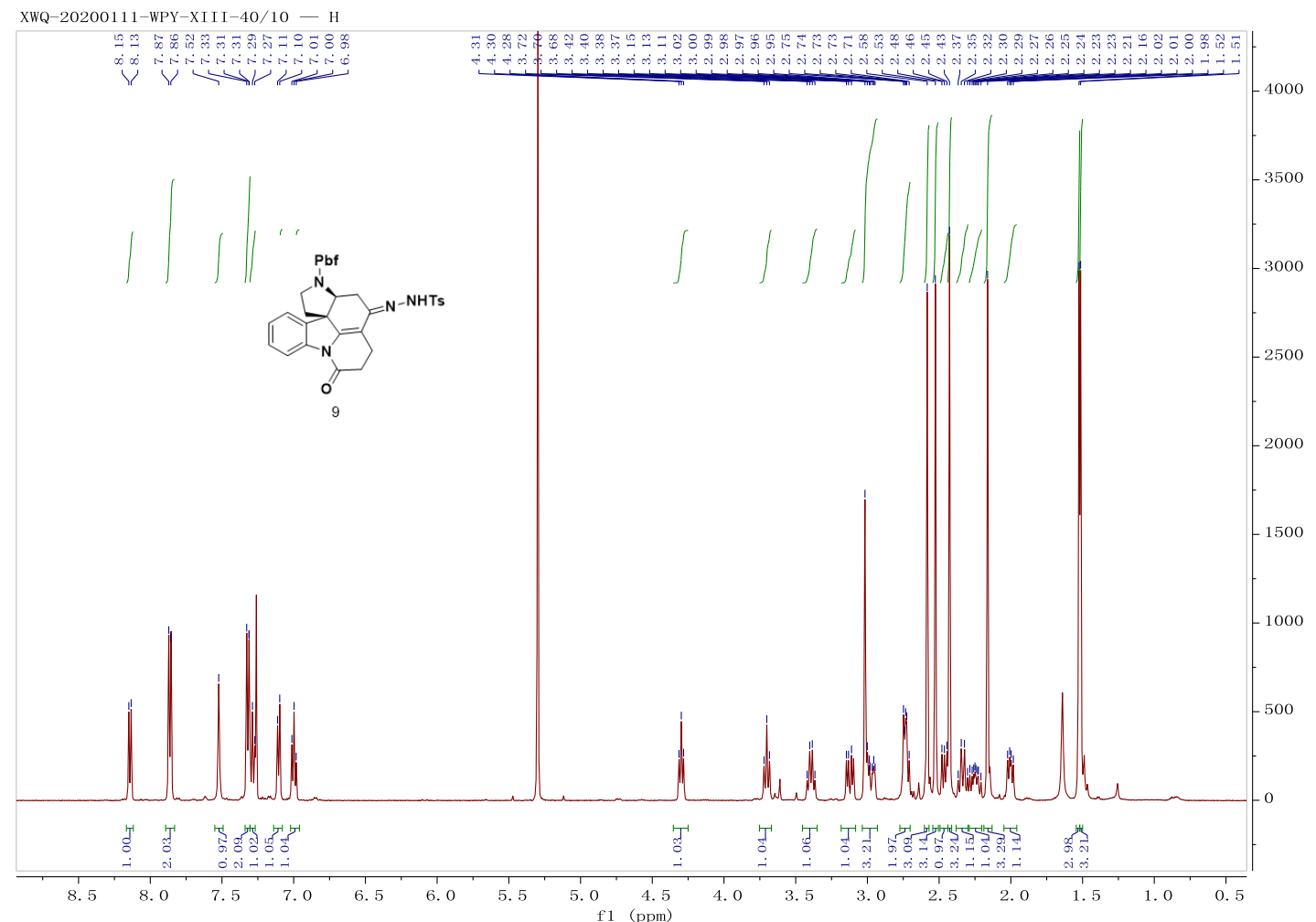

${ }^{13} \mathrm{C}$ NMR Spectrum of $9\left(126 \mathrm{MHz}, \mathrm{CDCl}_{3}\right)$

XWQ-20190625-WPY-X-15/11 - C13CPD CDC13 \{D: \2019-1\} ZHL 16

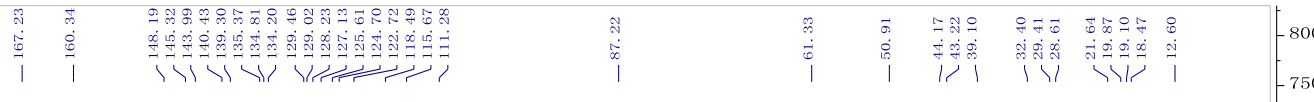

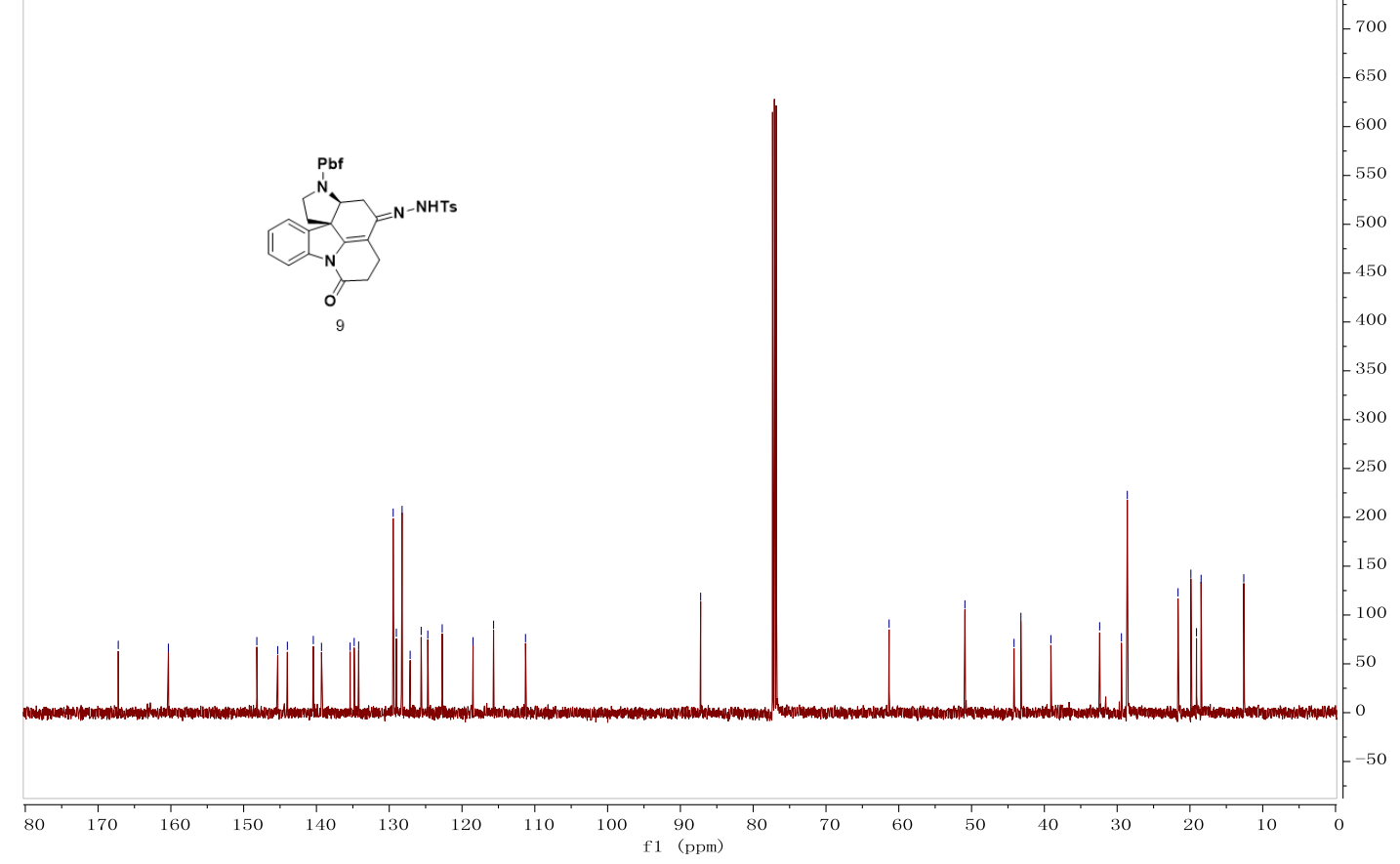




\section{${ }^{1} \mathrm{H}$ NMR Spectrum of $2\left(500 \mathrm{MHz}, \mathrm{CDCl}_{3}\right)$}

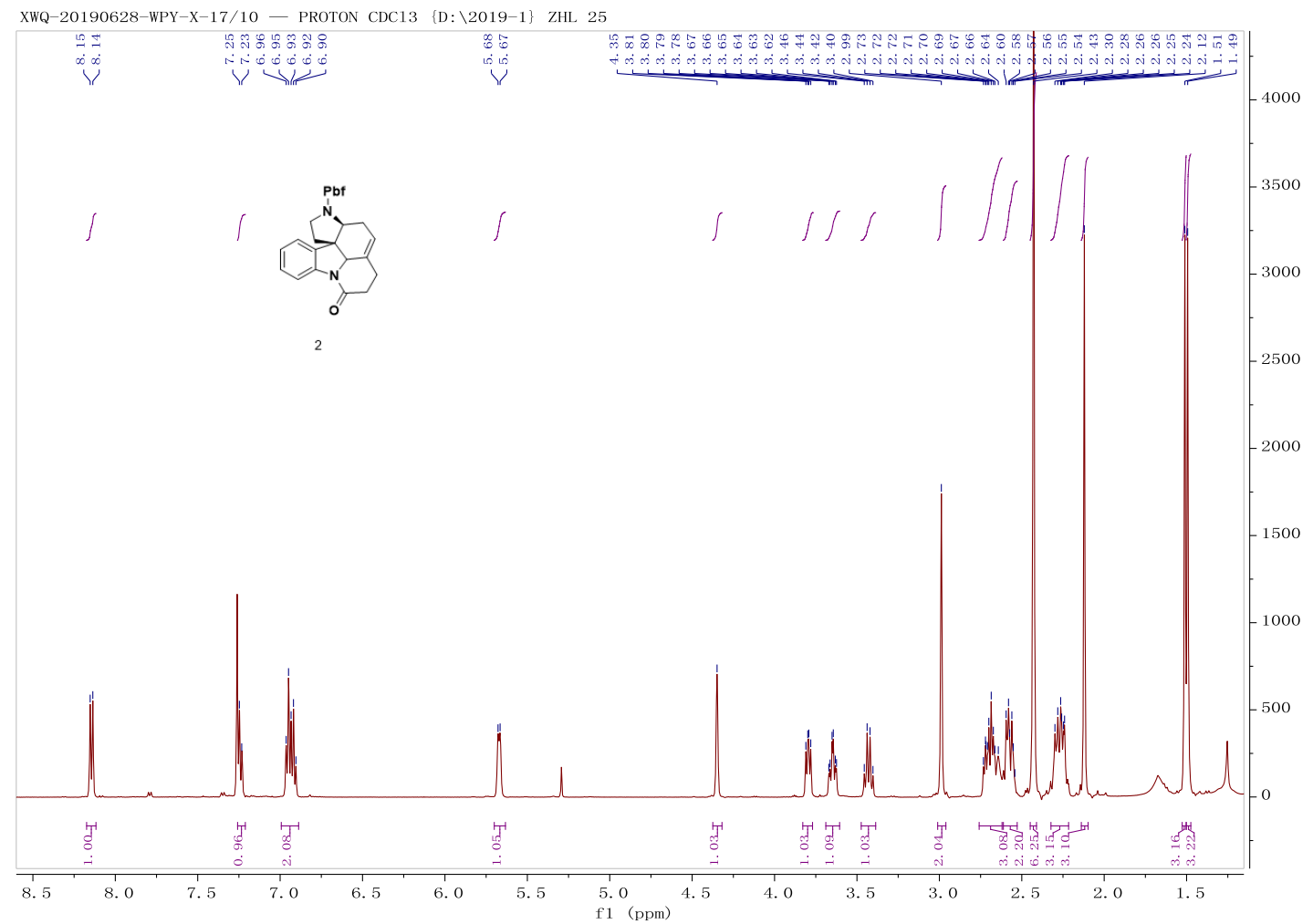

${ }^{13} \mathrm{C}$ NMR Spectrum of $2\left(126 \mathrm{MHz}, \mathrm{CDCl}_{3}\right)$

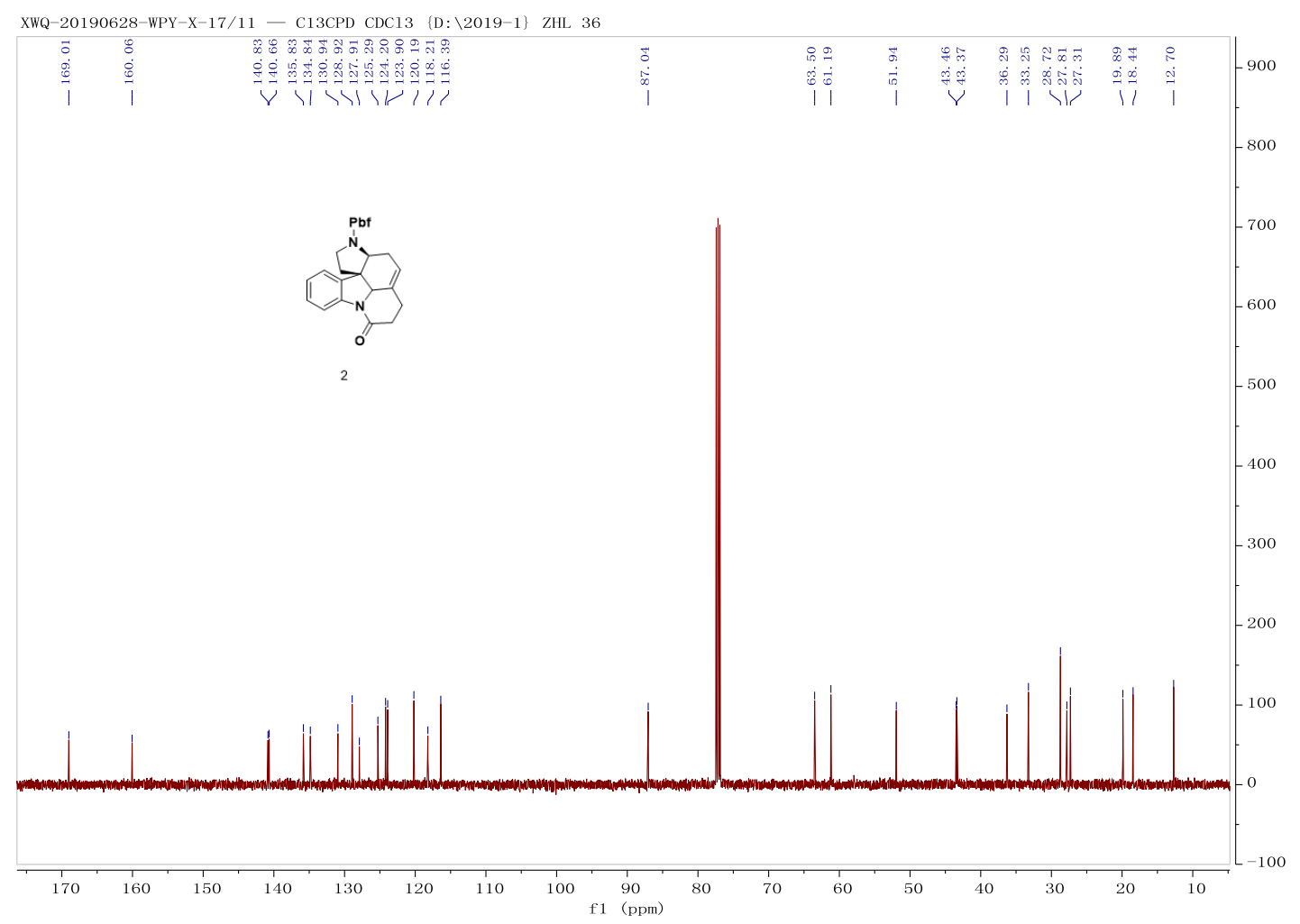




\section{${ }^{1} \mathrm{H}$ NMR Spectrum of $1\left(500 \mathrm{MHz}, \mathrm{CDCl}_{3}\right)$}

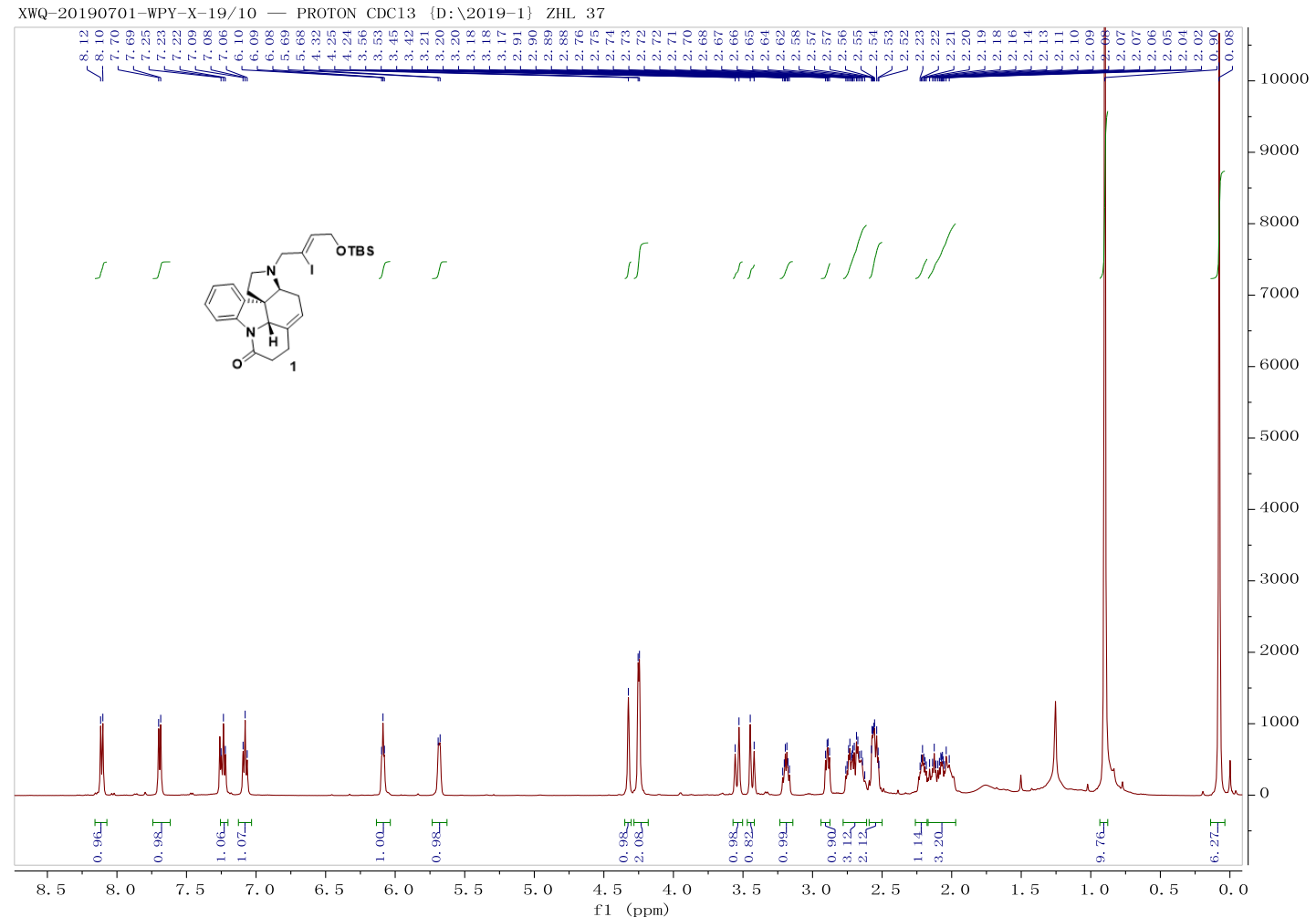

${ }^{13} \mathrm{C}$ NMR Spectrum of $1\left(126 \mathrm{MHz}, \mathrm{CDCl}_{3}\right)$

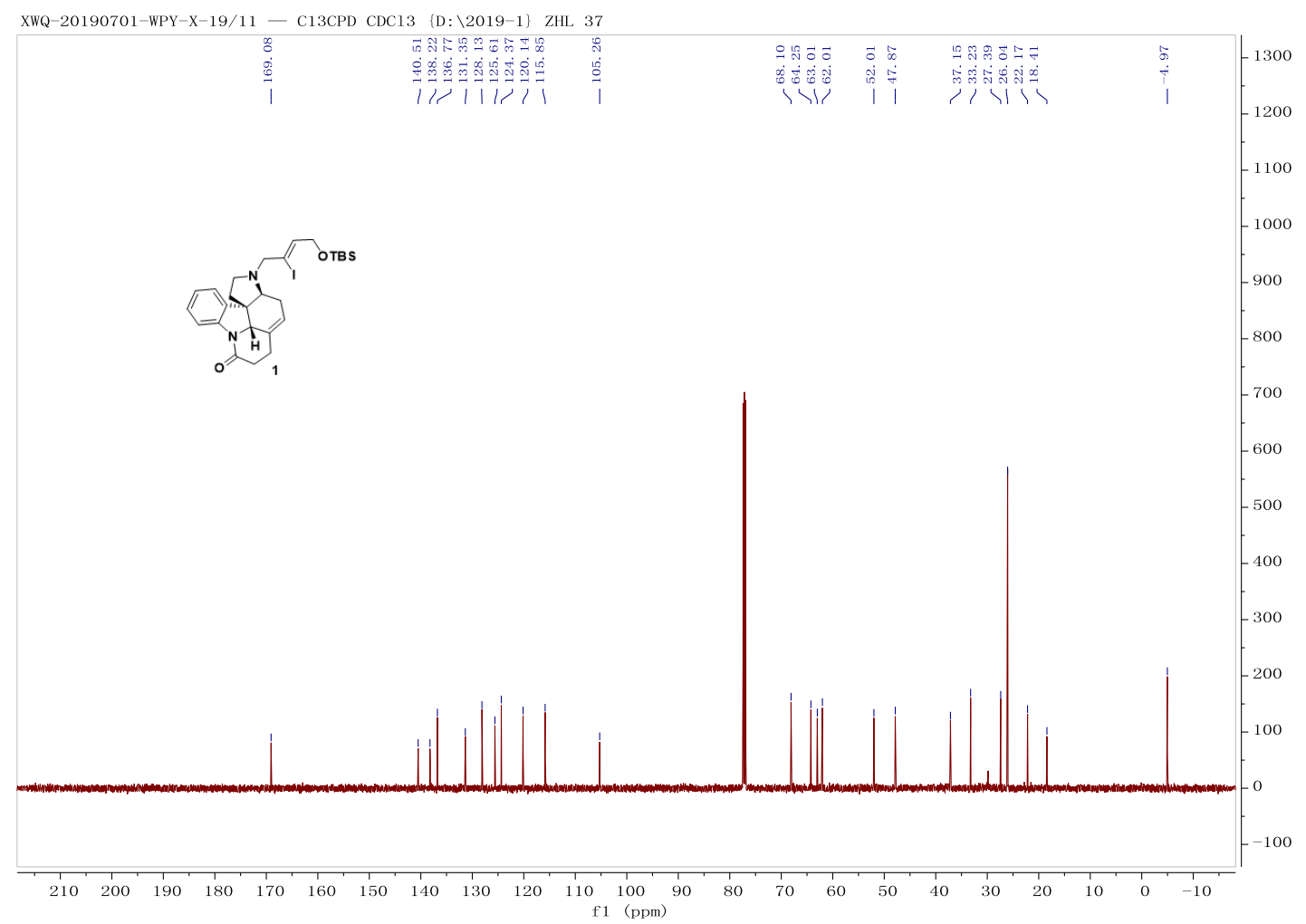




\section{${ }^{1} \mathrm{H}$ NMR Spectrum of $11\left(500 \mathrm{MHz}, \mathrm{CDCl}_{3}\right)$}

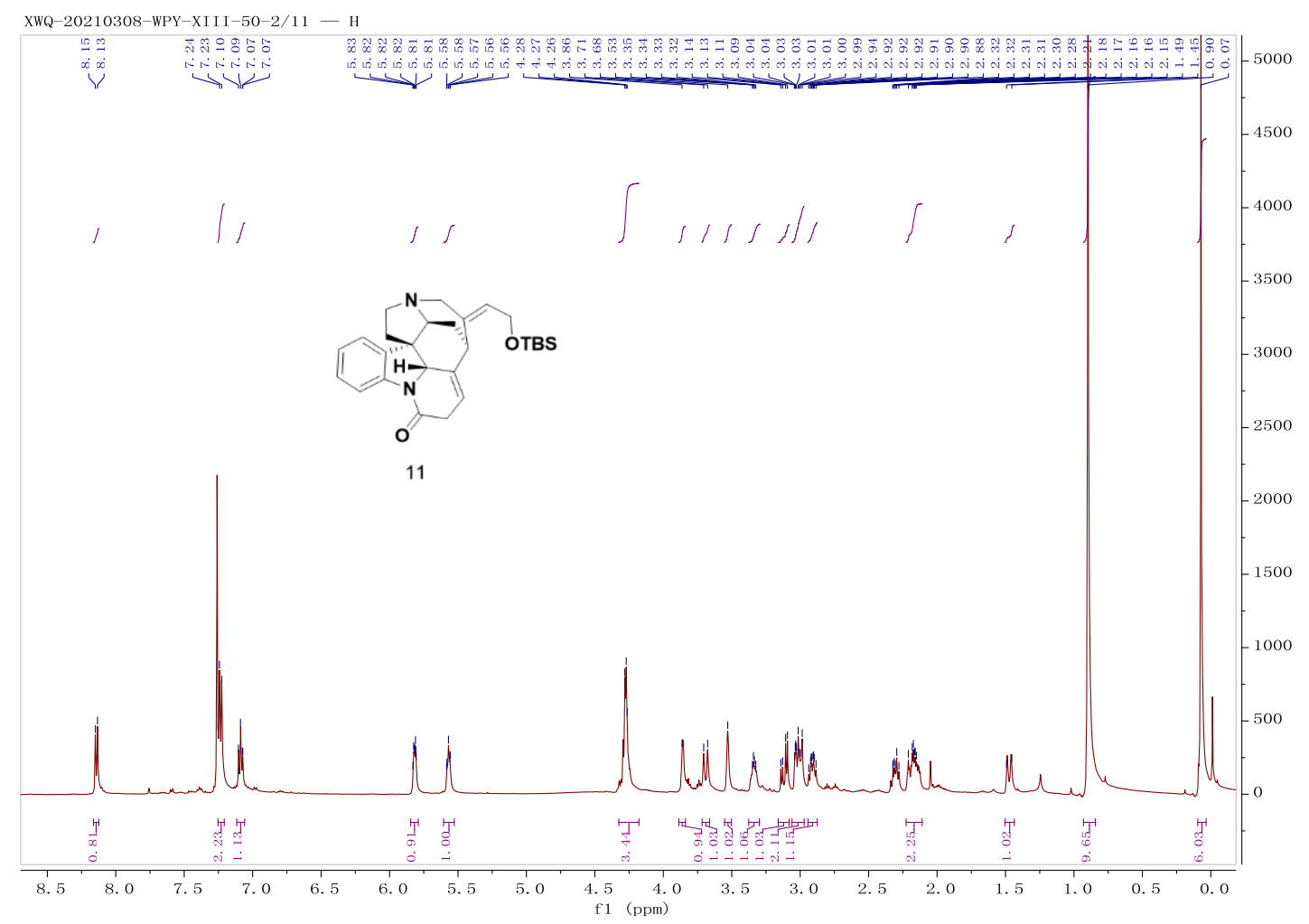

${ }^{13} \mathrm{C}$ NMR Spectrum of $11\left(126 \mathrm{MHz}, \mathrm{CDCl}_{3}\right)$

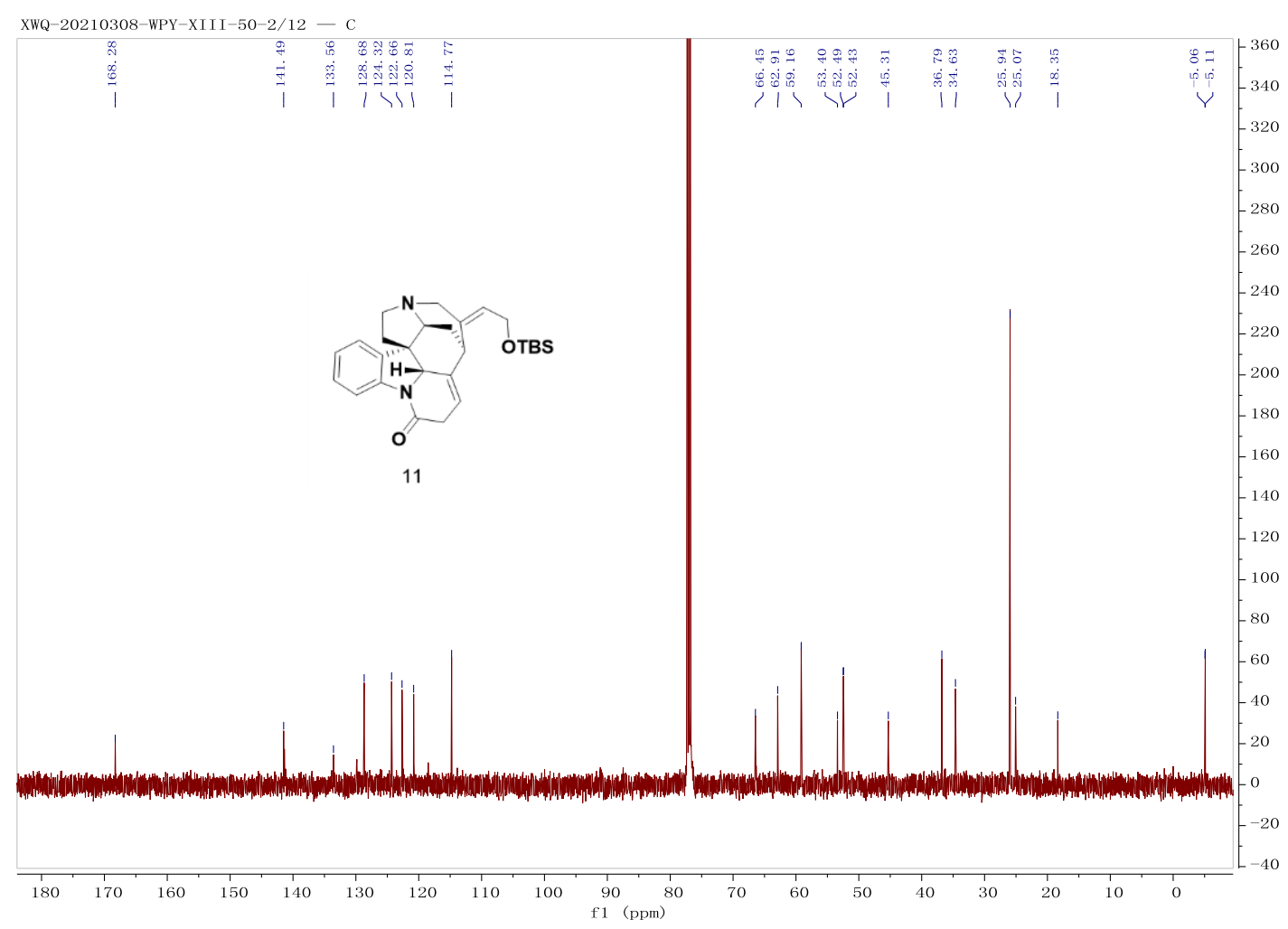




\section{${ }^{1} \mathrm{H}$ NMR Spectrum of (+)-Isostrychnine (500 MHz, $\left.\mathrm{CDCl}_{3}\right)$}

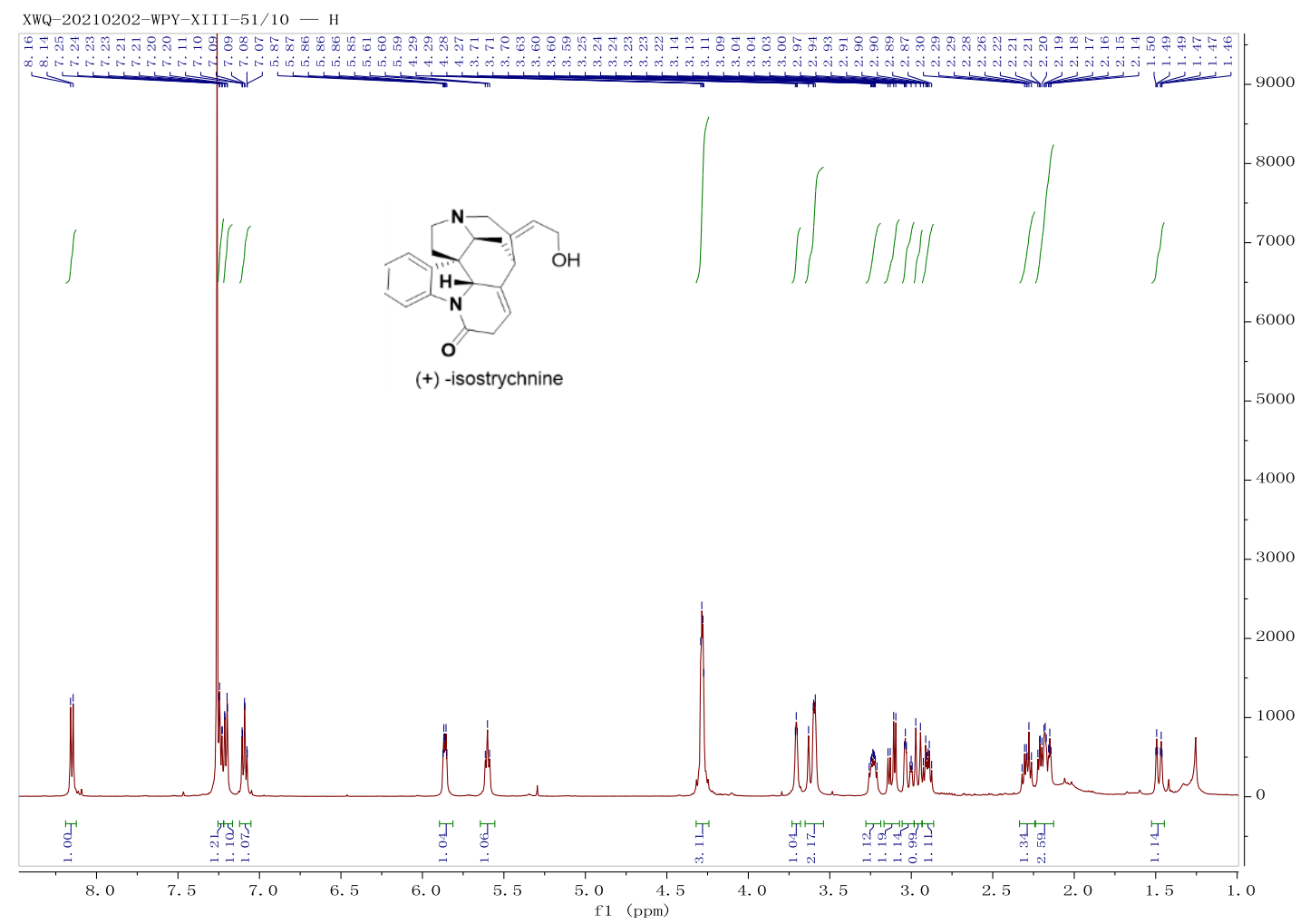

${ }^{13} \mathrm{C}$ NMR Spectrum of (+)-Isostrychnine (126 MHz, $\left.\mathrm{CDCl}_{3}\right)$

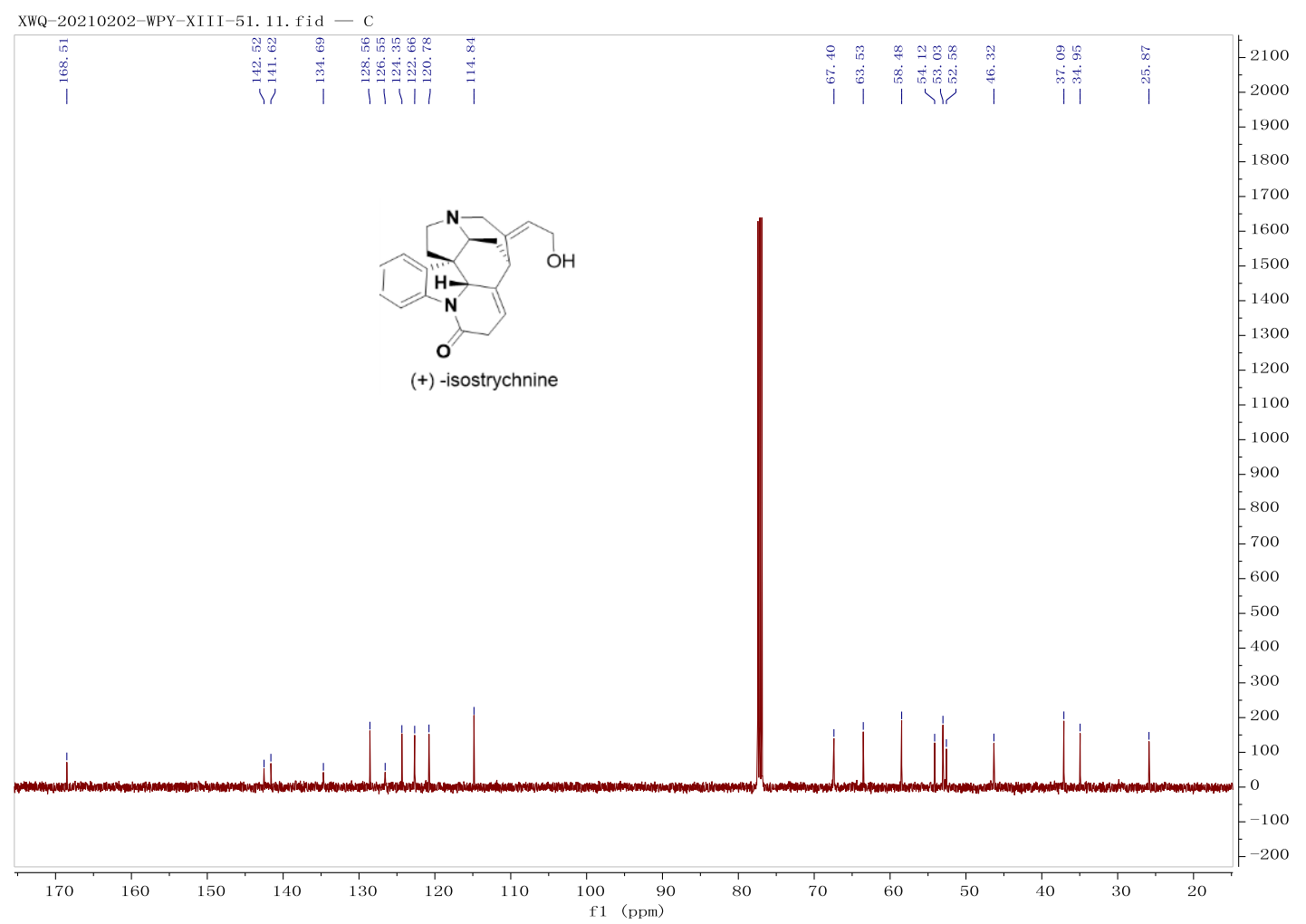

